\title{
Can Words Get in the Way? The Effect of Deliberation in Collective Decision Making
}

\section{Matias laryczower}

Princeton University

\section{Xiaoxia Shi}

University of Wisconsin-Madison

\section{Matthew Shum}

Caltech

\begin{abstract}
We quantify the effect of deliberation on the decisions of US appellate courts. We estimate a model in which strategic judges communicate before casting their votes and then compare the probability of mistakes in the court with deliberation with a counterfactual of no communication. The model has multiple equilibria, and preferences and information parameters are only partially identified. We find that there is a range of parameters in the identified set-when judges tend to disagree ex ante or their private information is imprecise-in which deliberation can be beneficial; otherwise, deliberation reduces the effectiveness of the court.
\end{abstract}

\section{Introduction}

Deliberation is an integral part of collective decision making. Instances of voting in legislatures, courts, boards of directors, and academic committees are generally preceded by some form of communication among

We are grateful to Maria Goltsman, Navin Kartik, Alessandro Lizzeri, Adam Meirowitz, Greg Pavlov, Alejandro Robinson Cortes, Leeat Yariv, and seminar participants at Colum-

Electronically published March 7, 2018

[ Journal of Political Economy, 2018, vol. 126, no. 2]

(c) 2018 by The University of Chicago. All rights reserved. $0022-3808 / 2018 / 12602-0006 \$ 10.00$ 
its members, ranging from free to fully structured, and from public to private or segmented. Does deliberation lead to better collective decisions? Or is deliberation among committee members detrimental to effective decision making?

On the face of it, the answer to this question seems straightforward: when committee members have the opportunity to talk with one another, they can share their private information and reach a better collective decision. Consider a situation in which jurors can vote in a straw poll before the actual voting takes place (as in Coughlan [2000]). When jurors' preferences are sufficiently similar, there is an equilibrium in which all members communicate their private information in the straw poll and then vote unanimously in the binding vote in favor of the posterior-preferred alternative. In this context, public communication can lead to an efficient outcome.

More generally, however, free-range communication among committee members might instead be detrimental to decision making, as individuals attempt to manipulate the beliefs of other committee members to achieve better outcomes for themselves. In fact, as we will see, allowing arbitrary communication possibilities among committee members can lead to worse outcomes than what would be obtained were members to vote without deliberation. Because of the ambiguity of the theoretical results, evaluating the effect of deliberation on outcomes becomes an empirical question, the answer to which depends on committee members' traits and on the equilibrium strategies they play in the data.

In this paper we quantify the effect of deliberation on collective choices in the context of criminal cases decided in the US courts of appeals. Our empirical strategy is to structurally estimate a model of voting with deliberation. This approach allows us to disentangle committee members' preferences, information, and strategic considerations and, ultimately, to compare equilibrium outcomes under deliberation with a counterfactual scenario in which prevote communication is precluded.

We accommodate prevote deliberation among judges by considering communication equilibria of the game (Forges 1986; Myerson 1986; Gerardi and Yariv 2007). Because the incentive for any individual member to convey her information truthfully depends on her expectations about how

bia, Emory, Erasmus University's Workshop in Political Economy 2012, Higher School of Economics (Russia), New York University, Princeton, Seoul National, Singapore Management, Sogang, Stanford (Stanford Institute for Theoretical Economics 2012), University College London, Chicago, Montréal, Penn, Western Ontario, and Washington University for comments. Financial support from National Science Foundation grants SES-1061326 (Iaryczower) and SES-1061266 (Shum) is gratefully acknowledged. We thank Alex Bolton, Benjamin Johnson, and Emerson Melo for excellent research assistance. Data are provided as supplementary material online. 
others will communicate, any natural model of deliberation will have a large multiplicity of equilibria, which leads to partial identification of the structural parameters characterizing judges' preferences and quality of information. Accordingly, we estimate an identified set for these parameters - a set of values of judges' preference and information parameters that are consistent with a mixture of equilibria generating the observed vote distribution - using a two-step procedure that allows flexibly for characteristics of the alternatives and the individuals.

The fundamental goal of this paper is to evaluate the effect of deliberation. To do this we compare outcomes that would emerge with and without deliberation. In particular, we focus on the probability that the court reaches an incorrect decision. The US appellate courts' task is to determine whether or not the law was applied correctly in the trial court. Thus, the appellate court reaches a wrong decision when it overturns a correct decision by the trial court or when it upholds an incorrect decision by the trial court.

Our results show that deliberation can reduce the probability of incorrect decisions when judges' preferences are sufficiently heterogeneous or their private information is relatively imprecise. In particular, we find that for a prior belief close to the frequency of overturning in the data, the probability of mistakes in communication equilibria consistent with the data is lower than in equilibria of the voting game without deliberation. In contrast, we find that prevote communication increases the prevalence of mistakes in the court when judges' preferences are not too heterogeneous and when their private information is relatively precise. In other words, deliberation can help for those points in the identified set for which individuals tend to disagree ex ante and cannot do too well if voting independently; otherwise, it tends to reduce the effectiveness of the court.

There are three parts to this result. First, prevote communication has the potential to lead to bad equilibria, where the court fails to use the private information of its members to its advantage. In these equilibria, judges vote against their own information because they infer during committee deliberation that the information of other judges contradicts their own. Second, in order to be consistent with the data, more heterogeneous courts also have to be "better," in the sense that judges must have more precise information and, for any given level of quality, must shed the worse equilibria. In other words, heterogeneous courts can be rationalized as generating the observed voting data, but only if they are competent and play equilibria in which they use their information effectively.

The third and final component is performance in the counterfactual of no deliberation. This has two parts. First, we find that for preference and information parameters in the estimated identified set, the set of equilibrium outcomes of the voting game without deliberation are generally 
close to the best outcomes that can be achieved in any communication equilibrium, including equilibria not consistent with the voting data. Thus the maximum potential gain from deliberation is generally low. Second, voting without deliberation performs worst when judges' private information is imprecise or when the court is very heterogeneous. It is in these instances that deliberation has a relatively large potential to improve outcomes. The potential appears to be realized in our data. The comparison shows that communication can, in fact, improve outcomes in these regions. However, it generally leads to higher error rates when judges are like-minded or when the quality of their private information is not too low, regardless of judges' prior beliefs.

The rest of the paper is organized as follows. Section II contains institutional background and also summary statistics from the data that describe key features of the US appellate courts. In Section III, we present a model of deliberative voting for appellate courts. Identification and estimation are discussed in Section IV. Section V presents the results. We present conclusions in Section VI. Proofs and supplementary materials are in the appendix, available online.

\section{US Appellate Courts: Data and Background}

In our empirical analysis of deliberation, we focus on criminal decisions in the US appellate courts. The appellate court setting is attractive for this analysis for two reasons. First, courts of appeals are small committees, composed of only three judges. This allows us to capture relevant strategic considerations in a relatively simple environment. Second, within each circuit, judges are assigned to panels and cases on an effectively random basis. The random assignment norm minimizes the impact of "case selection" whereby appellants are more likely to pursue cases in courts composed of more sympathetic judges.

The data are drawn together from two sources. The main source is the United States Courts of Appeals Data Base (Songer 2008). This provides detailed information about a substantial sample of cases considered by courts of appeals between 1925 and 1996, including characteristics of the cases, the judges hearing the case, and their votes. Among the roughly 16,000 cases in the full database, we restrict our attention to criminal cases, which make up around 20 percent of the total. The case and judge-specific variables we use in our analysis are summarized in table A.1 of the supplemental appendix. Additional information for judges involved in these decisions was obtained from the Multi-User Data Base on the Attributes of U.S. Appeals Court Judges (Zuk, Barrow, and Gryski 2009).

For each case, we include a dummy variable ("FedLaw") for whether the case is prosecuted under federal (rather than state) law, as well as dummy variables for the crime in each case. These crime categories are based on 
the nature of the criminal offense in the case and do not exhaust the set of possible crimes, but instead constitute "common" issues, bundling a relatively large number of cases within each label. Thus "aggravated" contains murder, aggravated assault, and rape cases. "White-collar" crimes include tax fraud and violations of business regulations and so forth. "Theft" includes robbery, burglary, auto theft, and larceny. The "narcotics" category encompasses all drug-related offenses. In addition to the nature of the crime, we also include information about the major legal issue under consideration in the appeal. In particular, we distinguish issues of jury instruction, sentencing, admissibility, and sufficiency of evidence from other legal issues.

We also include three variables that describe the makeup of the judicial panel deciding each case: an indicator for whether the panel is a Republican majority ("Republican Majority"), whether the panel contains at least one woman ("Woman on Panel"), and whether there is a majority of Harvard or Yale Law School graduates on the panel ("Harvard-Yale Majority"). This latter variable is included to capture possible "club effects" in voting behavior; the previous literature has pointed out how graduates from similar programs may share common judicial views and vote as a bloc.

Finally, we include four judge-specific covariates: "Republican" indicates a judge's affiliation to the Republican Party; "Yearsexp" that a judge has served on the court of appeals at the time that he or she decides a particular case (this variable varies both across judges and across cases); and "Judexp" and "Polexp," which measure the number of years of judicial and political experience of a judge prior to his or her appointment to the appellate court.

Since we are modeling the voting behavior on appellate panels, we distinguish between judges' votes for upholding $(v=0)$ versus overturning $(v=1)$ the decision of a lower court. Thus, given the majority voting rule, among the eight possible vote profiles, there are four that lead to an outcome of upholding the lower court's decision- $(0,0,0),(1,0,0),(0,1$, $0)$, and $(0,0,1)$ - and four leading to overturning- $(1,1,1),(1,1,0),(1$, $0,1)$, and $(0,1,1)$. In table A.1 in the supplemental appendix, we provide summary statistics for case and judge characteristics broken down by the four categories of vote outcomes (Unanimous to Overturn, Unanimous to Uphold, Divided to Overturn, and Divided to Uphold). As the table shows, the average case and judge characteristics vary substantially between the four vote outcomes, suggesting considerable variation in our data set.

Institutional features and preliminary data analysis.-An important component in any model of decision making in the courts of appeals is the information structure facing judges. The literature has essentially considered two approaches to modeling information in voting models: private- 
values "ideological" models and common-value models. Because of the relevance of this assumption in our analysis, here we explore this issue from an empirical standpoint. A consideration of the institutional background and formal statistical tests suggest that we model the appellate court as a common-values environment. ${ }^{1}$

First, the formal description of the appellate courts' task corresponds closely to a model in which common values are predominant. The appeals process in the US federal judicial system grants a losing party in a decision by a trial court (or district court) the right to appeal the decision to a federal court of appeals. The 94 federal judicial districts are organized into 12 regional circuits, each of which has a court of appeals. The appellate court's task is "to determine whether or not the law was applied correctly in the trial court." In fact, in order to win, the appellant "must show that the trial court made a legal error that affected the decision in the case" (http://www.uscourts.gov).

A purely ideological model of decision making in the courts seems simply unappealing in this environment, as it would imply that judges are unresponsive to information regarding errors in the trial court. In contrast, a common-values model captures the fact that judges' decisions are based on whether a given situation - about which the judges have imperfect information - occurred or not. Importantly, the notion of right and wrong we consider is whether the appeals court correctly or incorrectly determined that the law was applied correctly in the trial court; that is, the state variable in the model is not interpreted as guilt or innocence of the defendant but rather as whether the law was or was not applied correctly in the trial court. ${ }^{2}$

We complement the institutional perspective with a formal statistical test that exploits the random assignment of judges to cases in the US courts of appeals. Our test builds on two observations. First, given ran-

\footnotetext{
${ }^{1}$ In our framework, we assume that appellate judges' decisions are not linked over time, conditional on the case and judge-specific characteristics. This abstracts away from "career concerns," through which judges' behavior might change over time in response to incentives in the judicial career trajectory. To gauge the importance of career concerns, we report in table A. 2 of the supplemental appendix the differences in means for previous political experience, previous judicial experience, and years of experience in the court between dissenting vs. nondissenting judges, where dissenting judges are the ones who voted differently from the majority. The results show that the experience variables do not appear to be significantly different across dissenting judges and nondissenting judges, which suggests that career concerns may not be an important determinant of behavior, at least in the subset of cases we study.

${ }^{2}$ Our underlying assumption is then that if all relevant facts and law were known, judges would reasonably agree on whether the law was or was not applied correctly in the trial court. We believe that the US criminal appellate process and the kinds of reasoning required of an appellate court make this a reasonable assumption. In Sec. V.E we discuss this in more detail, paying particular attention to the distinction between questions of fact and questions of law, and provide a robustness exercise that focuses on a restricted sample of cases.
} 
dom assignment of judges to cases, judges' preferences could vary across cases and judges but should not vary with the characteristics of the other members of the panel given observable case characteristics. Second, in the standard ideological model considered in political economy, ajudge's vote should similarly not be correlated with his or her panel members' characteristics given observable case characteristics. ${ }^{3}$ This suggests a simple test for private versus interdependent values: we can regress a given judge's vote on case observables, the judge's own observables, and the other judges' observables. If the other judges' variables are significant, we reject the private-values hypothesis.

The presumption that judges are assigned to panels in an effectively random manner follows from an examination of institutional details in the court. The particular assignment procedures vary from circuit to circuit, with some circuits using explicitly random assignments (via random number generators) and others incorporating additional factors as dictated by practical considerations (e.g., availability), but the general intent is to not manipulate the assignment of judges to cases: "Judge assignment methods vary. The basic considerations in making assignments are to assure equitable distribution of caseloads and avoid judge shopping. By statute, the chief judge of each district court has the responsibility to enforce the court's rules and orders on case assignments. Each court has a written plan or system for assigning cases. The majority of courts use some variation of a random drawing" (http://www.uscourts.gov/Common/FAQS .aspx).

To assess the validity of random assignment among the cases in our data set, in table 1 we report coefficients from regressions of judge characteristics on case covariates. Random assignment should imply that case covariates have little predictive power on judge characteristics. Indeed, the results in table 1 show almost no statistically significant coefficients. None of the regressions has overall significance at even the 10 percent level, confirming that judges in these cases appear to be assigned randomly.

Having established the random assignment of judges to cases, we move on to the test proper. We implement this test by running linear regressions on our data, in which the outcome variable is whether a given judge voted to overturn $(=1)$ or uphold $(=0)$, and the explanatory variables include characteristics of the other judges on the committee. Table 2 shows the regression under several specifications. We find that the private-values hypothesis is indeed rejected: across all the specifications, we find strong evidence that the makeup of a committee does affect the voting behavior of committee members; across all specifications, the committee variables are (jointly) significant in an $F$-test with a $p$-value less than 8 percent.

\footnotetext{
${ }^{3}$ This would not be the case if judges had intertemporal agreements (e.g., logrolling), as it could be the case in legislatures, but these agreements seem unusual in our context.
} 
TABLE 1

Random Assignment? Regressions of Judge on Case Characteristics

\begin{tabular}{llccccc}
\hline \hline & \multicolumn{5}{c}{ Average Judge Characteristics } \\
\cline { 2 - 7 } Case Covariates & Republican & Yrsexp & Judexp & Polexp & Nonwhite & Female \\
\hline Federal & $-.024^{*}$ & $.386^{*}$ & .044 & -.004 & .005 & .006 \\
Aggravated & $-.038^{* *}$ & .313 & $.381^{+}$ & -.194 & -.000 & .007 \\
White-collar & -.007 & -.203 & .029 & .143 & -.008 & -.004 \\
Theft & -.015 & .184 & -.153 & .128 & -.007 & .003 \\
Narcotics & $-.024^{+}$ & .057 & $-.298^{+}$ & .080 & -.000 & -.002 \\
Jury instruction & -.005 & .060 & -.084 & .082 & -.002 & .005 \\
Sentencing & -.012 & -.189 & -.054 & -.078 & $.013^{*}$ & .002 \\
Admissibility & $-.018^{*}$ & -.039 & $.267^{*}$ & -.038 & -.005 & .006 \\
Sufficency of evidence & .006 & .218 & .111 & .008 & .002 & $-.009 * *$ \\
Overall significance $(p$-value $)$ & .194 & .630 & .258 & .390 & .397 & .205 \\
\hline
\end{tabular}

NoTE.-All regressions include constant terms (not reported for brevity) and fixed effects for the interactions of year $\times$ circuit. Overall significance is the $p$-value of the $F$-test for the joint significance of all case characteristics. Sample size $=3,239$.

+ Significant at the 15 percent level.

* Significant at the 10 percent level.

** Significant at the 5 percent level.

*** Significant at the 1 percent level.

As discussed above, this evidence supports our modeling of the appellate court scenario as one involving interdependent values rather than involving independent private values. Accordingly, in the next section, we introduce a common-value specification that is prominent in the existing literature on committee voting and deliberation (Austen-Smith and Banks 1996; Feddersen and Pesendorfer 1997, 1998) and deliberation (Coughlan 2000; Austen-Smith and Feddersen 2005, 2006; Gerardi and Yariv 2007) to address important policy and design counterfactuals raised in this literature, such as the informational benefits or efficiencies from deliberation. $^{4}$

\section{A Model of Voting in US Appellate Court Committees}

On the basis of the institutional features and data patterns discussed in the foregoing section, we map out a model of committee decision making and deliberation in appellate court panels. Our basic model builds on Feddersen and Pesendorfer (1998) by allowing for heterogeneous biases

\footnotetext{
${ }^{4}$ See Wan and Xu (2010), Grieco (2014), and Xu (2014) for analyses of nonvoting games with interdependent but non-common-value types. Stasser and Titus (1985), Fischman (2011, 2015), and Gole and Quinn (2014) consider non-common-values-based models of committee decision making in which agents may have noninformational or behavioral motives. The role of deliberation in such environments is less clear, and for that reason, we follow most of the existing deliberation literature and focus on common-value models in this paper.
} 
TABLE 2

Ordinary Least Squares Regressions of Vote on Case, Judge, and Committee Variables

\begin{tabular}{|c|c|c|c|c|}
\hline & $\begin{array}{c}\text { Specification } \\
1\end{array}$ & $\begin{array}{l}\text { Specification } \\
2\end{array}$ & $\begin{array}{l}\text { Specification } \\
3\end{array}$ & $\begin{array}{c}\text { Specification } \\
4\end{array}$ \\
\hline \multicolumn{5}{|l|}{$\begin{array}{l}\text { Committee variables } \\
\text { (sum of panel mates' } \\
\text { characteristics): }\end{array}$} \\
\hline Republican dummy & $-.017^{+}$ & & $-.017^{+}$ & \\
\hline $\begin{array}{l}\text { Political experience } \\
\text { (in decades) }\end{array}$ & $.024 *$ & $.026 *$ & & $.022 *$ \\
\hline Female dummy & -.050 & & $-.054^{*}$ & \\
\hline $\begin{array}{l}\text { Joint significance of } \\
\text { committee variables }\end{array}$ & & & & \\
\hline$(p$-value of $F$-test $)$ & $.048 * *$ & $.064 *$ & $.076^{*}$ & $.050 * *$ \\
\hline \multicolumn{5}{|l|}{ Control variables: } \\
\hline Judge characteristics & Yes & Yes & Yes & No \\
\hline Case observables & Yes & Yes & Yes & Yes \\
\hline $\begin{array}{l}\text { Circuit } \times \text { year fixed } \\
\text { effects }\end{array}$ & Yes & Yes & Yes & Yes \\
\hline
\end{tabular}

Note. - Case variables controlled include dummies for crime types (federal, aggravate, white-collar, theft, and narcotics) and dummies for reason of appeal (jury instruction, sentencing, admissibility, and sufficiency of evidence). Judge characteristics controlled include dummies for Republican, nonwhite, and female, as well as three experience measures (appeals court experience, judicial experience, and political experience). Significance levels are computed with errors clustered by both judge and committee. We use the two-way clustering procedure in Cameron et al. (2011). Sample size $=9,717$.

+ Significant at the 15 percent level.

* Significant at the 10 percent level.

** Significant at the 5 percent level.

*** Significant at the 1 percent level.

and quality of information (all of which are public information). To this we add deliberation as in Gerardi and Yariv (2007), considering communication equilibria. An attractive and powerful rationale for focusing on the set of communication equilibria is that the set of outcomes induced by communication equilibria coincides with the set of outcomes induced by sequential equilibria of any cheap talk extension of the voting game. ${ }^{5}$

There are three judges, $i=1,2,3$. Judge $i$ votes to uphold $\left(v_{i}=0\right)$ or overturn $\left(v_{i}=1\right)$ the decision of the lower court. The decision of the court, $v \in\{0,1\}$, is that of the majority of its members. That is, if we let $\vec{v}$ denote the vector $\left(v_{1}, v_{2}, v_{3}\right)^{\prime}$ (written as $v_{1} v_{2} v_{3}$ below whenever it does not cause confusion) and let the court's decision be denoted $v=\psi(\vec{v})$, then $\psi(\vec{v})=1$ if and only if $\sum_{i} v_{i} \geq 2$.

In line with our previous discussion, we assume that there is a correct decision in each case, which is modeled as a hidden state variable $\omega \in\{0,1\}$

${ }^{5}$ Coughlan (2000) and Austen-Smith and Feddersen $(2005,2006)$ introduce an alternative approach in this context, extending the voting game with one round of public deliberation. For other models of deliberation, see Li, Rosen, and Suen (2001), Doraszelski, Gerardi, and Squintani (2003), Meirowitz (2006), Landa and Meirowitz (2009), and Lizzeri and Yariv (2011). 
reflecting whether errors have been committed at trial $(\omega=1)$ or not $(\omega=$ $0)$. The realization of this random variable $\omega$ in any given case is only imperfectly observed by the judges in the court of appeals.

Judge $i$ suffers a cost $\pi_{i} \in(0,1)$ when the court incorrectly overturns the lower court $(v=1$ when $\omega=0)$ and of $1-\pi_{i}$ when it incorrectly upholds the lower court $(v=0$ when $\omega=1)$. The payoffs of $v=\omega=0$ and $v=\omega=1$ are normalized to zero. Thus given information $\mathcal{I}$, judge $i$ votes to overturn if and only if $\operatorname{Pr}^{i}(\omega=1 \mid \mathcal{I}) \geq \pi_{i}$. Accordingly, $\pi_{i}$ can be thought of as the hurdle imposed by judge $i$ on the amount of information that must be available about facts constituting errors in trial for her to be willing to overturn the decision of the lower court. Thus, $\pi_{i}>1 / 2$ reflects a positive hurdle (a bias toward upholding), while $\pi_{i}<1 / 2$ reflects a negative hurdle (a bias toward overturning). ${ }^{6}$

Confronted with a case, each appellate judge has common prior beliefs $\rho \equiv \operatorname{Pr}(\omega=1)$ and observes a private signal $t_{i} \in\{0,1\}$ for $i=1$, 2, 3 that is imperfectly correlated with the truth; that is, $\operatorname{Pr}\left(t_{i}=k \mid \omega=\right.$ $k)=q_{i}>1 / 2$ for $k=0,1$. The parameter $q_{i}$ captures the informativeness of $i$ 's signals. ${ }^{7}$ The judges' signals are independent from each other conditional on $\omega$. For convenience, we let $\theta \equiv(p, \vec{q})$.

In the absence of deliberation, this setting describes a voting $G$, as in Feddersen and Pesendorfer (1998). We extend this model to allow for prevote deliberation among the judges, that is, for judges to discuss the case with each other and potentially to reveal their private information to each other. Following Gerardi and Yariv (2007), we model deliberation by considering equilibria of an extended game in which judges exchange messages after observing their signals and before voting. In particular, we consider a cheap talk extension of the voting game that relies on a fictional mediator, who helps the judges communicate. In this augmented game, judges report their signals $\vec{t}$ to the mediator, who then selects the vote profile $\vec{v}$ with probability $\mu(\vec{v} \mid \vec{t})$ and informs each judge of her own vote. The judges then vote. A communication equilibrium is a sequential equilibrium of this cheap talk extension in which judges (i) convey their private information truthfully to the mediator and (ii) follow the mediator's recommendations in their voting decisions. ${ }^{8}$ These define two sets

\footnotetext{
${ }^{6}$ In the estimation, we will allow the biases $\pi_{i}$ of each judge $i$ to vary with case-specific and individual-specific characteristics. The biases that judges can have in any given type of case can reflect a variety of factors, inducing a nonneutral approach to this case, such as ingrained theoretical arguments about the law, personal experiences, or ideological considerations.

${ }^{7}$ We assume $q_{i}>1 / 2$ without loss of generality, because if $q_{i}<1 / 2$, we can redefine the signal as $1-t_{i}$. The assumption that the signal quality does not depend on $\omega$ is made only for simplicity.

${ }^{8}$ Note that in equilibrium players do not necessarily infer the information available to the mediator. Thus, the requirement that players report truthfully to the mediator does not imply that players will report truthfully to the other players in a given unmediated communication protocol implementing the same outcomes.
} 
of incentive compatibility conditions, which we formally describe next as the "deliberation stage" and "voting stage" constraints, respectively.

Voting stage.-At the voting stage, private information has already been disclosed to the mediator. Still the equilibrium probability distributions $\mu(\cdot \mid \vec{t})$ over vote profiles $\vec{v}$ must be such that each judge $i$ wants to follow the mediator's recommendation $v_{i}$. Hence we need that for all $i=1,2,3$, for all $v_{i} \in\{0,1\}$, and for all $t_{i} \in\{0,1\}$,

$$
\sum_{t_{-i}} p\left(t_{-i} \mid t_{i} ; \theta\right) \sum_{v_{-i}}\left[u_{i}\left(\psi\left(v_{i}, v_{-i}\right), \vec{t}\right)-u_{i}\left(\psi\left(1-v_{i}, v_{-i}\right), \vec{t}\right)\right] \mu(\vec{v} \mid \vec{t}) \geq 0,
$$

where as usual $t_{-i} \equiv\left(t_{j}, t_{k}\right)$ and $v_{-i} \equiv\left(v_{j}, v_{k}\right)$ for $j, k \neq i$. Here $p\left(t_{-i} \mid t_{i} ; \theta\right)$ denotes the conditional probability mass function of $t_{-i}$ given $t_{i}$, and $u_{i}(\psi(\vec{v}), \vec{t})$ denotes the utility of judge $i$ when the decision is $\psi(\vec{v})$ and the signal profile is $\vec{t}$. Note that $u_{i}\left(\psi\left(v_{i}, v_{-i}\right), \vec{t}\right)-u_{i}\left(\psi\left(1-v_{i}, v_{-i}\right), \vec{t}\right)=$ 0 whenever $v_{-i} \notin \operatorname{Piv}^{i} \equiv\left\{\left(v_{j}, v_{k}\right): v_{j} \neq v_{k}\right\}$. Then (1) is equivalent to (2) (for $v_{i}=1$ ) and (3) (for $v_{i}=0$ ) for $i=1,2,3$ and for all $t_{i} \in\{0,1\}$ :

$$
\sum_{t_{-i}} p\left(t_{-i} \mid t_{i} ; \theta\right)\left[p_{\omega}(1 \mid \vec{t} ; \theta)-\pi_{i}\right] \sum_{v_{-i} \in \mathrm{Piv}^{i}} \mu\left(1, v_{-i} \mid \vec{t}\right) \geq 0
$$

and

$$
\sum_{t_{-i}} p\left(t_{-i} \mid t_{i} ; \theta\right)\left[\pi_{i}-p_{\omega}(1 \mid \vec{t} ; \theta)\right] \sum_{v_{-i} \in \mathrm{Piv}^{i}} \mu\left(0, v_{-i} \mid \vec{t}\right) \geq 0,
$$

where $p_{\omega}(\omega \mid \vec{t} ; \theta)$ denotes the conditional probability mass function of $\omega$ given $\vec{t}$. There are therefore 12 such equilibrium conditions at the voting stage.

Deliberation stage.-At the deliberation stage, communication equilibria require that judges are willing to disclose their private information truthfully to the mediator, anticipating the outcomes induced by the equilibrium probability distributions $\mu(\cdot \mid \vec{t})$ over vote profiles $\vec{v}$. This includes ruling out deviations at the deliberation stage that are profitable when followed up by further deviations at the voting stage. To consider this possibility we define the four "disobeying" strategies:

$$
\begin{aligned}
& \tau_{1}\left(v_{i}\right)=v_{i}: \\
& \tau_{2}\left(v_{i}\right)=1-v_{i}: \\
& \text { always obey } \\
& \tau_{3}\left(v_{i}\right)=1: \\
& \tau_{4}\left(v_{i}\right)=0: \\
& \text { always disobey } \\
& \text { always overturn } \\
& \text { always uphold }
\end{aligned}
$$

We require that for all $i=1,2,3$, all $t_{i} \in\{0,1\}$, and $\tau_{j}(\cdot), j=1,2,3,4$ :

$$
\begin{gathered}
\sum_{t_{-i}} p\left(t_{-i} \mid t_{i} ; \theta\right) \sum_{v}\left[u_{i}(\psi(\vec{v}), \vec{t}) \mu\left(\vec{v} \mid t_{i}, t_{-i}\right)\right. \\
\left.-u_{i}\left(\psi\left(\tau_{j}\left(v_{i}\right), v_{-i}\right), \vec{t}\right) \mu\left(\vec{v} \mid 1-t_{i}, t_{-i}\right)\right] \geq 0 .
\end{gathered}
$$


There are therefore 24 such equilibrium conditions at the deliberation stage.

For any given $(\theta, \vec{\pi})$, the conditions (2), (3), and (4) characterize the set of communication equilibria $M(\theta, \vec{\pi})$; that is,

$$
M(\theta, \vec{\pi})=\{\mu \in \mathcal{M}:(\theta, \vec{\pi}, \mu) \text { satisfies (2), (3), and (4) }\},
$$

where $\mathcal{M}$ is the set of all possible values that $\mu$ can take, and it can be conveniently thought of as the set of $8 \times 8$-dimensional matrices whose elements lie in $[0,1]$ and each row sums to one. Note that $M(\theta, \vec{\pi})$ is convex, as it is defined by linear inequality constraints on $\mu$.

REMARK 1 (Robust communication equilibria). Note that for given $v_{i}$, the vote profiles in which the other judges vote unanimously to overturn or uphold do not enter the incentive compatibility conditions at the voting stage. Thus, without any additional refinement, the set of communication equilibria includes strategy profiles in which some members of the court vote against their preferred alternative only because their vote cannot influence the decision of the court. These include not only strategy profiles $\mu$ that put positive probability only on unanimous votes but also profiles in which $i$ votes against her preferred alternative only because conditional on her signal and her vote recommendation she is surebelieves with probability one-that her vote is not decisive. Consider the example in table 3 .

The strategy profile in table 3 is a communication equilibrium for $\rho=$ 0.1 , and $\pi_{i}=0.3, q_{i}=0.6$ for $i=1,2,3$. However, judge 1 votes to overturn with positive probability even if $\operatorname{Pr}(\omega=1 \mid \vec{t})<\pi$ for all $\vec{t}$, in spite of the fact that nonunanimous vote profiles are played with positive probability. The result arises because conditional on $t_{1}=0$ (cols. 5-8)

TABLE 3

A Nonrobust Communication Equilibrium

\begin{tabular}{lcccccccc}
\hline \hline & \multicolumn{7}{c}{ Signal Profile } \\
\cline { 2 - 8 } Vote Profile & $(1,0,0)$ & $(1,0,1)$ & $(1,1,0)$ & $(1,1,1)$ & $(0,0,0)$ & $(0,0,1)$ & $(0,1,0)$ & $(0,1,1)$ \\
\hline$(1,0,0)$ & .000 & .033 & .000 & .015 & .005 & .000 & .077 & .000 \\
$(1,0,1)$ & .000 & .000 & .000 & .000 & .000 & .000 & .000 & .000 \\
$(1,1,0)$ & .000 & .000 & .000 & .000 & .000 & .000 & .000 & .000 \\
$(1,1,1)$ & .119 & .282 & .132 & .274 & .216 & .118 & .202 & .132 \\
$(0,0,0)$ & .855 & .657 & .859 & .689 & .623 & .850 & .688 & .858 \\
$(0,0,1)$ & .000 & .000 & .000 & .000 & .131 & .000 & .000 & .000 \\
$(0,1,0)$ & .026 & .027 & .010 & .022 & .025 & .031 & .033 & .009 \\
$(0,1,1)$ & .000 & .000 & .000 & .000 & .000 & .000 & .000 & .000 \\
$\operatorname{Pr}(v=1 \mid t)$ & .119 & .282 & .132 & .274 & .216 & .118 & .202 & .132 \\
$\operatorname{Pr}(v=0 \mid t)$ & .881 & .718 & .868 & .726 & .784 & .882 & .798 & .868 \\
$\operatorname{Pr}(\omega=1 \mid t)$ & .069 & .143 & .143 & .273 & .032 & .069 & .069 & .143 \\
\hline
\end{tabular}

Note.-The equilibrium is for $\rho=0.1$ and $\pi_{i}=0.3 ; q_{i}=0.6$ for $i=1,2,3$. For each row $\vec{v}$ and column $\vec{t}$, the entry gives $\mu(\vec{v} \mid \vec{t})$. 
and $v_{1}=1$ (rows $\left.1-4\right)$, judge 1 believes that either $\vec{v}=(1,0,0)$ or $\vec{v}=(1$, $1,1)$ are played. As a result, her vote is not decisive in equilibrium, and judge 1 is willing to vote to overturn. The same is true in this example conditional on $t_{1}=1$. A similar logic holds for judges 2 and 3 .

Because these equilibria are not robust to small perturbations in individuals' beliefs about how others will behave, we rule them out. To do this, we require that each individual best responds to beliefs that are consistent with small trembles (occurring with probability $\eta$ ) on equilibrium play, so that all vote profiles have positive probability after any signal profile. Formally, in all equilibrium conditions - at both the voting and deliberation stages - we substitute $\operatorname{Pr}(\vec{v} \mid \vec{t})$ in place of $\mu(\vec{v} \mid \vec{t})$, where for any $\vec{t}$ and $\vec{v}$,

$$
\operatorname{Pr}(\vec{v} \mid \vec{t})=\sum_{\overrightarrow{\hat{v}}}\left[\mu(\overrightarrow{\hat{v}} \mid \vec{t}) \prod_{j=1}^{3}(1-\eta)^{\hat{v}_{j}=v_{j}} \eta^{\hat{v}_{j} \neq v_{j}}\right] .
$$

The $\eta$ we use in the empirical section is $0.01 .^{9}$

Adverse communication.--Having eliminated nonrobust equilibria, we know that judges' voting decisions will reflect their posterior beliefs after deliberation. In fact, provided that

$$
\sum_{t_{-i}} \sum_{v_{-i} \in \mathrm{Piv}^{i}} \mu\left(\left(1, v_{-i}\right) \mid\left(t_{i}, t_{-i}\right)\right) \operatorname{Pr}\left(t_{-i} \mid t_{i} ;(\vec{q}, \rho)\right)>0,
$$

the conditions (2) can be written as

$$
\operatorname{Pr}\left(\omega=1 \mid v_{i}=1, t_{i}, \operatorname{Piv}^{i} ;(\vec{q}, \rho, \mu)\right) \leq \pi_{i} ;
$$

that is, conditional on her vote $v_{i}$, signal $t_{i}$, and on being pivotal in the court's decision, judge $i$ prefers to overturn the decision of the lower court. Similarly conditions (3) boil down to $\operatorname{Pr}\left(\omega=1 \mid v_{i}=0, t_{i}, \operatorname{Piv}^{i} ;(\vec{q}, \rho, \mu)\right) \geq$ $\pi_{i}$. It follows that if communication is to lead to inferior outcomes, it has to be through judges' beliefs after deliberation. The question then is, How much can deliberation influence rational judges' beliefs?

As it turns out, the answer is "quite a lot." We illustrate this with an example. Let $\pi_{1}=0.25, \pi_{2}=\pi_{3}=0.6$, and $\rho=1 / 2$ and suppose that $q_{i}=$ 0.90 for $i=1,2,3$; that is, judge 1 is biased toward overturning the lower court, while judges 2 and 3 are biased toward upholding the decisions of the lower court, and judges have uninformative priors and relatively accurate private information. Table 4 describes a particular communication equilibrium $\tilde{\mu} .^{10}$ This equilibrium is of interest here because it leads to incorrect decisions with high probability, even when $q=0.9$. Consider

\footnotetext{
${ }^{9}$ To evaluate the robustness of our results, we replicate the analysis for $\eta=0.001$ and $\eta=$ 0.000001 . The results are qualitatively unchanged.

${ }^{10}$ As in table 3 , the cell corresponding to row $\vec{v}$ and column $\vec{t}$ gives the equilibrium probability that $\vec{v}$ is played given a signal realization $\vec{t}$, i.e., $\mu(\bar{v} \mid \vec{t})$. Thus, for example, $\mu(100 \mid$ 100) $=0.044$.
} 
TABLE 4

Equilibrium Consistent with the Data with Large Error Rates

\begin{tabular}{lccccccccc}
\hline \hline \multirow{2}{*}{$\begin{array}{l}\text { Vote } \\
\text { Profile }\end{array}$} & \multicolumn{7}{c}{ Signal Profile } \\
\cline { 2 - 10 } & $(1,0,0)$ & $(1,0,1)$ & $(1,1,0)$ & $(1,1,1)$ & $(0,0,0)$ & $(0,0,1)$ & $(0,1,0)$ & $(0,1,1)$ & $\operatorname{Pr}(v)$ \\
\hline$(1,0,0)$ & .044 & .110 & .321 & .000 & .000 & .066 & .023 & .000 & .025 \\
$(1,0,1)$ & .000 & .023 & .141 & .002 & .006 & .006 & .029 & .160 & .019 \\
$(1,1,0)$ & .073 & .003 & .044 & .008 & .000 & .003 & .081 & .003 & .012 \\
$(1,1,1)$ & .187 & .115 & .080 & .254 & .256 & .252 & .150 & .028 & .223 \\
$(0,0,0)$ & .351 & .604 & .403 & .736 & .739 & .537 & .433 & .735 & .676 \\
$(0,0,1)$ & .082 & .021 & .000 & .000 & .000 & .136 & .170 & .000 & .018 \\
$(0,1,0)$ &. $\mathbf{2 6 3}$ & .000 & .011 & .000 & .000 & .000 & .113 & .000 & .017 \\
$(0,1,1)$ & .000 & .124 & .000 & .000 & .000 & .000 & .000 & .074 & .009 \\
$\operatorname{Pr}(v=1 \mid t)$ & .255 & .259 & .259 & .258 & .256 & .255 & .255 & .259 & \\
$\operatorname{Pr}(\omega=1 \mid t)$ & .100 & .900 & .900 & .999 & .001 & .100 & .100 & .900 & \\
$\operatorname{Pr}(t)$ & .045 & .045 & .045 & .365 & .365 & .045 & .045 & .045 & \\
\hline
\end{tabular}

Note.-The equilibrium is for $q=0.90, \pi_{1}=0.25$, and $\pi_{2}=\pi_{3}=0.6$.

for example column 2, corresponding to $\vec{t}=101$. While the probability that the decision should be overturned given $\vec{t}=101$ is fairly large - that is, $\operatorname{Pr}(\omega=1 \mid \vec{t}=101))=.9$-in equilibrium the court overturns when $\vec{t}=101$ roughly one-fourth of the times: $\operatorname{Pr}(v=1 \mid \vec{t}=101)=.26$.

To understand how this happens, consider the problem of judge 1 . Note that judge 1 is predisposed to overturn, as $\pi_{1}=0.25$. Nevertheless, in equilibrium she sometimes votes to uphold, even after observing a signal that errors have been made in trial. Now, consider judge 1's equilibrium inference when in equilibrium she votes to uphold $\left(v_{1}=0\right)$, given that she received a signal in favor of overturning, $t_{1}=1$. Because she is supposed to vote zero, judge 1 can rule out (put probability zero on) the vote profile $\vec{v}=100$ and thus the entire first row of the matrix. Similarly, she can rule out rows 2,3 , and 4 . Because she knows that she received a 1 signal, she can rule out the possibility that $\vec{t}=000$ (col. 5). Similarly, she can rule out columns 6,7 , and 8 . Because only events in which she is pivotal to the decision are payoff consequential, she can rule out $\vec{v}=(0,0,0)$ and $\vec{v}=(0,1,1)$ (rows 5 and 8$)$. We are thus left with the bold cells in the table. But this indicates that the posterior probability that the other two judges received a 0 signal is considerably high. In fact, $\operatorname{Pr}\left(t_{2}=0, t_{3}=0 \mid v_{1}=0, t_{1}=1, \operatorname{Piv}^{1}\right)$ is given by

$$
\begin{gathered}
\frac{[\mu(001 \mid 100)+\mu(010 \mid 100)] \operatorname{Pr}(\vec{t}=100)}{\sum_{\left(t_{2}, t_{3}\right)}\left[\mu\left(001 \mid 1, t_{2}, t_{3}\right)+\mu\left(010 \mid 1, t_{2}, t_{3}\right)\right] \operatorname{Pr}\left(\vec{t}=\left(1, t_{2}, t_{3}\right)\right)} \\
=\frac{(0.082+0.263) 0.045}{0.017}=.915 .
\end{gathered}
$$

Thus, the equilibrium inference about the information of other judges ends up overwhelming her own private information, leading to a posterior probability that errors were not committed at trial (i.e., should up- 
hold) of only.17. (The same logic applies to judges 2 and 3, leading to a posterior probability of exactly. 60 for both judges 2 and 3, consistent with equilibrium.)

The example illustrates that after all incentive constraints are taken into account, deliberation can still have a powerful effect on the beliefs of rational, fully Bayesian judges. The result has the flavor of the results of Kamenica and Gentzkow (2011), albeit in a different strategic setting. The two games have many differences, of course, as here there are three privately informed players, who are both senders and receivers of information, while Kamenica and Gentzkow consider a two-player game, where an uninformed sender can choose the information service available to a single decision maker. Crucially, however, choosing a communication equilibrium $\mu$ effectively entails choosing an information service for each judge (as receiver) subject to the equilibrium constraints assuring that each player reports its information truthfully (to the mediator). While a communication equilibrium adds constraints to manipulation of beliefs, the example illustrates that there is still room for players to persuade one another through deliberation.

The fact that deliberation can lead to better or worse outcomes than the corresponding game without deliberation implies that quantifying the effect of deliberation is ultimately an empirical question. In the next sections we develop an empirical framework that allows us to tackle this issue.

\section{From Model to Data}

The structural estimation of voting models with incomplete information is a relatively recent endeavor in empirical economics. This paper extends several recent papers examining voting behavior in committees with incomplete information and common values (Iaryczower and Shum 2012a, 2012b; Hansen, McMahon, and Velasco Rivera 2013; Iaryczower, Lewis, and Shum 2013). ${ }^{11}$ In those papers committee members are assumed to vote without deliberating prior to the vote. This paper takes the analysis one step further by allowing explicitly for communication among judges. As we show below, this extension is far from a trivial one, as the deliberation stage introduces multiple equilibria, rendering the conventional estimation approach inapplicable.

\footnotetext{
${ }^{11}$ Iaryczower, Katz, and Saiegh (2013) use a similar approach to study information transmission among chambers in the US Congress. For structural estimation of models of voting with private values and complete information, see Poole and Rosenthal (1985, 1991), Heckman and Snyder (1997), Londregan (1999), and Clinton, Jackman, and Rivers (2004) for the US Congress and Martin and Quinn (2002, 2007) for the US Supreme Court. Degan and Merlo (2009), De Paula and Merlo (2009), and Henry and Mourifie (2013) consider nonparametric testing and identification of the ideological voting model.
} 
In terms of estimation and inference, this paper draws on recently developed tools from the econometric literature on partial identification (e.g., Chernozhukov, Hong, and Tamer 2007; Beresteanu, Molchanov, and Molinari 2011). In a closely related paper, Kawai and Watanabe (2013) study the partial identification of a strategic voting model using aggregate vote share data from Japanese municipalities. ${ }^{12}$

\section{A. Partial Identification of Model Parameters}

The immediate goal of the estimation is to recover the signal/state distribution parameters, $\theta$, and the judges' preference vector $\vec{\pi}$. The information used to recover these parameters is the distribution of the voting profiles, $p_{v}(\vec{v})$, which can be identified from the data. Here we define the sharp identified set for the model parameters. ${ }^{13}$ The sharp identified set of $\{\theta, \vec{\pi}\}$ is the set of parameters that can rationalize $p_{v}(\vec{v})$ under some equilibrium selection mechanism $\lambda$-a mixing distribution over $\mu \in M(\theta, \vec{\pi})$. In other words, the sharp identified set $\mathcal{A}_{0}$ is the set of $(\theta, \vec{\pi}) \in \Theta \times[0,1]^{3}$ such that there exists a $\lambda$ that satisfies

$$
p_{v}(\vec{v})=\int_{\mu \in M(\theta, \vec{\pi})} \lambda(\mu) \sum_{\vec{t}} \mu(\vec{v} \mid \vec{t}) p(\vec{t} ; \theta) d \mu .
$$

However, because the set $M(\theta, \vec{\pi})$ of communication equilibria is convex, whenever there exists a mixture $\lambda$ satisfying (6), there exists a single equilibrium $\mu \in M(\theta, \vec{\pi})$ such that $p_{v}(\vec{v})=\sum_{\vec{t}} \mu(\vec{v} \mid \vec{t}) p(\vec{t} ; \theta) .{ }^{14}$ Thus $\mathcal{A}_{0}$ boils down to

$$
\mathcal{A}_{0}=\left\{(\theta, \vec{\pi}) \in \Theta \times[0,1]^{3}: \exists \mu \in M(\theta, \vec{\pi}) \text { s.t. } p_{v}(\vec{v})=\sum_{\vec{t}} \mu(\vec{v} \mid \vec{t}) p(\vec{t} ; \theta)\right\} .
$$

\footnotetext{
${ }^{12}$ While we are not aware of other papers analyzing deliberation with field data in a setting similar to the one considered here, some recent papers have analyzed deliberation in laboratory experiments. Guarnaschelli, McKelvey, and Palfrey (2000), using the straw poll setting of Coughlan (2000), show that subjects do typically reveal their signal (above 90 percent of subjects do so), but that contrary to the theoretical predictions, individuals' private information has a significant effect on their final vote. Goeree and Yariv (2011) show that when individuals can communicate freely, they typically disclose their private information truthfully and use public information effectively (as in Austen-Smith and Feddersen [2005], voters' bias parameters are private information, so individuals are identical ex ante). For other experimental results on deliberation, see McCubbins and Rodriguez (2006) and Dickson, Hafer, and Landa (2008).

${ }^{13}$ The sharpness of the identified set is in the sense of Berry and Tamer (2006), Beresteanu et al. (2011), and Galichon and Henry (2011). However, our estimation approach differs quite substantially from the approaches in those papers.

${ }^{14}$ This fact implies an observational equivalence between a unique communication equilibrium being played in the data and a mixture of such equilibria. Sweeting (2009) and De Paula and Tang (2012) discuss the nonobservational equivalence between mixture of equilibria and unique mixed-strategy equilibria in coordination games.
} 
We will also introduce the following set $\mathcal{B}_{0}$ :

$$
\mathcal{B}_{0}=\left\{(\theta, \vec{\pi}, \mu) \in \mathcal{B}: \mu \in M(\theta, \vec{\pi}) \text { and } p_{v}(\vec{v})=\sum_{\vec{t}} \mu(\vec{v} \mid \vec{t}) p(\vec{t} ; \theta)\right\} \text {, }
$$

where $\mathcal{B}=\Theta \times[0,1]^{3} \times \mathcal{M}$ and $\mathcal{M}$ is the set of $\mu 8 \times 8$-dimensional matrices, the elements of which are positive and each row sums to one. The set $\mathcal{B}_{0}$ is the sharp identified set of $\{\theta, \vec{\pi}, \mu\}$, where $\mu$ is the true mixture voting assignment probability. The identified set $\mathcal{A}_{0}$ can be considered as the projection of $\mathcal{B}_{0}$ onto its first $d_{\theta}+3$ dimensions, corresponding to the parameters $(\theta, \vec{\pi})$.

Identification in a symmetric model: intuition.-Before proceeding on to the estimation of the identified set, we provide some intuition for the identification of the model parameters by analyzing a stripped-down model in which the three judges are symmetric, in the sense that they have identical preferences and quality of information. That is, the preference parameters are identical across judges $\left(\pi_{1}=\pi_{2}=\pi_{3}=\pi\right)$ and so are the signal accuracies $\left(q_{1}=q_{2}=q_{3}=q\right)$. In this simple model, there are only three parameters $(\rho, q, \pi)$.

In figure 1 we show the pairs $(\pi, q)$ in the identified set for four different hypothetical vote profile vectors and given values of the common prior $\rho$. The figure on the upper-left panel plots the identified set for $\rho=0.5$ and a uniform distribution of vote profiles, that is, $p_{v}(\vec{v})=1 / 8$ for all $\vec{v}$. Because of the symmetry of the vote profile, the identified set is symmetric with respect to the $\pi=0.5$ line. Moreover, the set of preference parameters $\pi$ in the identified set for each value of $q$ is increasing in $q$. Thus, high-ability judges can be very predisposed toward either upholding (requiring considerably more information supporting that mistakes were made at trial to overturn) or overturning (requiring considerably more information supporting that mistakes were not made at trial to uphold) and still play equilibria consistent with the data. However, lowability judges must be highly malleable - willing to uphold or overturn even with little information that errors in trial have been committed or not - if they are to be consistent with the data.

The figure on the top right plots the pairs $(\pi, q)$ in the identified set for the uniform distribution over vote profiles and a prior probability of $\rho=0.1$ that mistakes were made at trial. Because the prior is very favorable toward upholding the decision of the lower court, only judges who are very biased toward overturning $(\pi \ll 1 / 2)$ — who require a high certainty that errors in trial have not been committed in order to upholdcan vote in a way consistent with the data. The reason is that for these types of judges, the opposite bias and priors compensate each other, effectively making them equivalent to a nonbiased judge with uniform priors over the state. On the other hand, judges who are already predisposed 

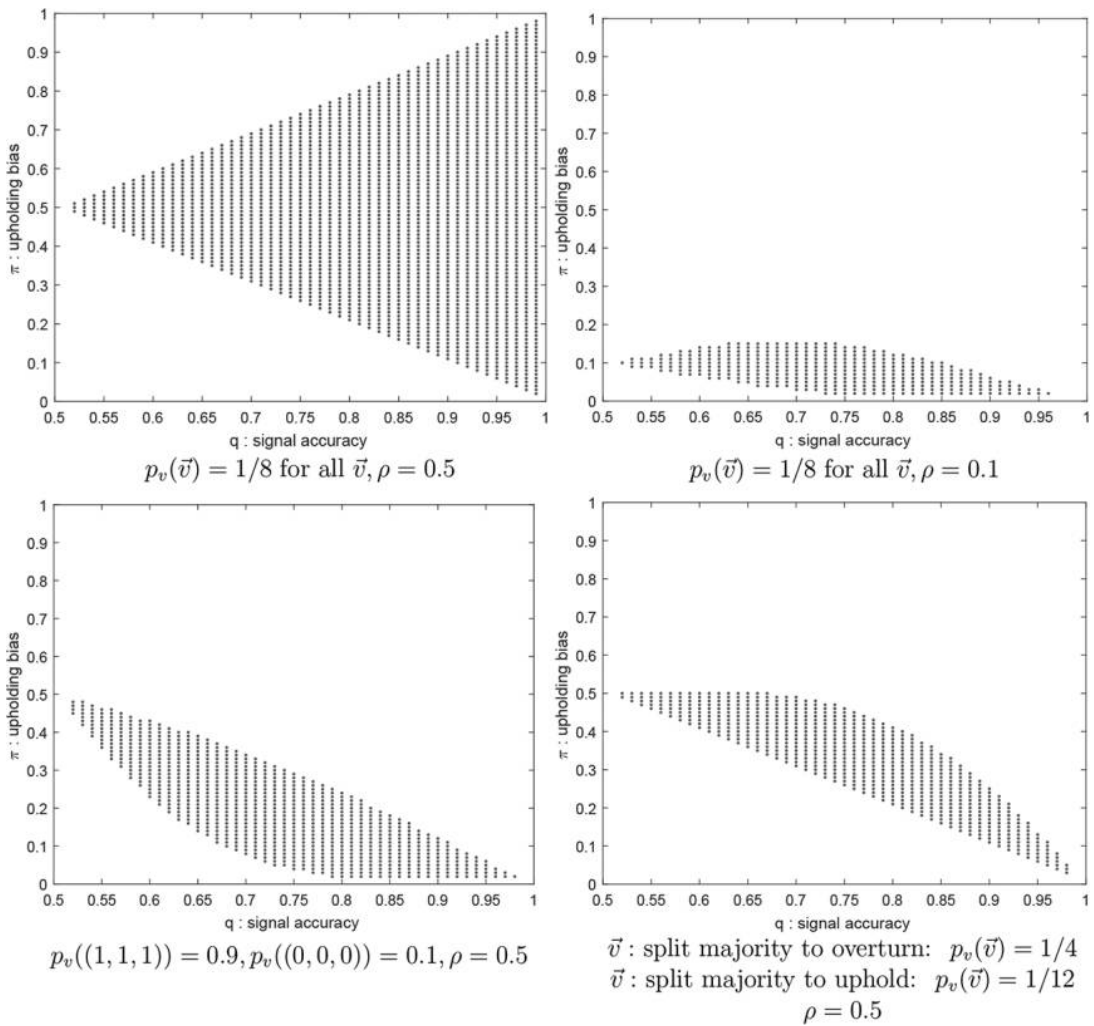

FIG. 1.-Identification of second-stage parameters: computational examples. Figures present computations from a simplified model in which the preference parameters are assumed identical across judges: $\pi_{i}=\pi$ for all $i$. We graph the identified set for the parameters $(\pi, q)$ under four different sets of the vote probabilities $p(\vec{v})$ and the prior parameter $\rho$. In each graph, the $x$-axis ranges over values of $q$ and the $y$-axis ranges over values of the common preference parameter $\pi$. Additional details and discussion are provided in the text. Color version available as an online enhancement.

toward upholding $(\pi \gg 1 / 2)$ become more extreme only once the prior is taken into consideration and thus are not inclined to vote to overturn.

The figures in the lower panel return to $\rho=0.5$ but consider nonuniform distributions of vote profiles. In the lower-left figure, only unanimous votes have positive probability, and the probability of overturning is $p_{v}(1,1,1)=.9$, while $p_{v}(0,0,0)=.1$. As in the first figure, low-ability judges must be willing to uphold or overturn with even little information that errors in trial have been committed or not if they are to be consistent with the data. However, high-ability judges must be biased toward overturning (must demand a high certainty that errors in trial have not been committed in order for them not to overturn), and increasingly so the higher the information precision. The same result holds in the 
lower-right figure, where also overturning is more likely, but only nonunanimous votes have positive probability. In this case, however, more malleable judges are consistent with the data for any given level of $q$. Note that in all figures, $\pi \rightarrow \rho$ as $q \rightarrow 1 / 2$. The reason is that as signals become less informative, in order to get a judge to vote for both alternatives some of the time, the judge has to be increasingly closer to being indifferent between voting one way or the other, after bias and prior beliefs are taken into consideration.

\section{B. Estimation}

To study the estimation of the identified set, we define the criterion function

$$
Q(\theta, \vec{\pi} ; W)=\min _{\mu \in M(\theta, \vec{\pi})} Q(\theta, \vec{\pi}, \mu ; W),
$$

where

$$
Q(\theta, \vec{\pi}, \mu, W)=\left(\vec{p}_{v}-P_{t}(\theta) \vec{\mu}\right)^{\prime} W\left(\vec{p}_{v}-P_{t}(\theta) \vec{\mu}\right)^{\prime},
$$

and where $\vec{p}_{v}=\left(p_{v}(111), p_{v}(110), p_{v}(101), p_{v}(100), p_{v}(010), p_{v}(001), p_{v}(000)\right)^{\prime}$, $\vec{\mu}$ is a 64 -vector whose $8 k+1$ st to $8 k+8$ th coordinates are the $(k+1)$ st row of $\mu(\vec{v} \mid \vec{t})$ for $k=0, \ldots, 7, P_{t}(\theta)=p(\vec{t}, \theta)^{\prime} \otimes\left[I_{7} \mid 0_{7}\right]$, and $W$ is a positive definite weighting matrix specified later.

We estimate the vote probabilities by the empirical frequencies of the vote profiles:

$$
\hat{p}_{v}(\vec{v})=\frac{1}{n} \sum_{l=1}^{n} 1\left(V_{l}=\vec{v}\right),
$$

where $V_{l}$ is the observed voting profile for case $l$ and $n$ is the sample size. Assuming that the cases are independent and identically distributed by the law of large numbers, $\hat{p}_{v}(\vec{v}) \rightarrow_{p} p_{v}(\vec{v})$ for all $\vec{v} \in \mathcal{V}$, where $\mathcal{V}=\{111$, $110,101,100,010,001,000\}$. One can define a sample analogue estimator for $\mathcal{A}_{0}$ :

$$
\hat{\mathcal{A}}_{n}=\underset{(\theta, \vec{\pi}) \in \Theta \times[0,1]^{3}}{\operatorname{argmin}} Q_{n}\left(\theta, \vec{\pi}, W_{n}\right),
$$

where $W_{n}$ is an estimator of $W$ and $Q_{n}$ is defined like $Q$ except with $\vec{p}_{v}$ replaced by its sample analogue $\vec{p}_{v}$. The set $\hat{\mathcal{A}}_{n}$ is the estimated identified set (EIS) that we compute using the data.

To compute $\hat{\mathcal{A}}_{n}$, one can follow steps below:

1. For each $(\theta, \vec{\pi})$, compute $Q_{n}\left(\theta, \vec{\pi} ; W_{n}\right)$ by solving the quadratic programming problem: 


$$
\begin{aligned}
Q_{n}\left(\theta, \vec{\pi} ; W_{n}\right)= & \min _{\vec{\mu} \in[0,1]^{64}}\left(\widehat{\vec{p}}_{v}-P_{t}(\theta) \vec{\mu}\right)^{\prime} W_{n}\left(\widehat{\vec{p}}_{v}-P_{t}(\theta) \vec{\mu}\right)^{\prime} \\
& \text { subject to }(2),(3), \text { and }(4), \\
& \sum_{j=k+1}^{k+8} \vec{\mu}_{j}=1, k=0, \ldots, 7 .
\end{aligned}
$$

2. Repeat step 1 for many grid points of $(\theta, \vec{\pi}) \in \Theta \times[0,1]^{3}$.

3. Form $\hat{\mathcal{A}}_{n}$ as the set of minimizers of $Q_{n}\left(\theta, \vec{\pi} ; W_{n}\right)$ among the grid points.

The following theorem establishes the consistency of $\hat{\mathcal{A}}_{n}$ with respect to the Hausdorff distance:

$$
\begin{array}{r}
d_{H}\left(\hat{\mathcal{A}}_{n}, \mathcal{A}_{0}\right)=\max \left\{\sup _{(\theta, \vec{\pi}) \in \hat{\mathcal{A}}_{n}} \inf _{\left.\theta^{*}, \vec{\pi}^{*}\right) \in \mathcal{A}_{0}}\left\|(\theta, \vec{\pi})-\left(\theta^{*}, \vec{\pi}^{*}\right)\right\|,\right. \\
\left.\sup _{\left(\theta^{*}, \vec{\pi}^{*}\right) \in \mathcal{A}_{0}} \inf _{(\theta, \vec{\pi}) \in \hat{\mathcal{A}}_{n}}\left\|(\theta, \vec{\pi})-\left(\theta^{*}, \vec{\pi}^{*}\right)\right\|\right\} .
\end{array}
$$

In general partially identified models, the sample analogue estimators for the identified sets are not typically consistent with respect to the Hausdorff distance (see, e.g., Chernozhukov et al. 2007). Our problem has a special structure that guarantees consistency under mild conditions.

Theorem 1. Suppose that $W_{n} \rightarrow_{p} W$ for some finite positive definite matrix $W$ and $\Theta$ is compact. Also suppose that $\operatorname{cl}\left(\operatorname{int}\left(\mathcal{B}_{E}\right) \cap \mathcal{B}_{0}\right)=\mathcal{B}_{0}$, where $\mathcal{B}_{E}=\{(\theta, \vec{\pi}, \mu) \in \mathcal{B}: \mu \in \mathcal{M}(\theta, \vec{\pi})\} .{ }^{15}$ Then $d_{H}\left(\hat{\mathcal{A}}_{n}, \mathcal{A}_{0}\right) \rightarrow_{p} 0$ as the sample size $n$ goes to infinity.

In the results below we will also consider the construction of confidence sets for the partially identified model parameters; we use the two-step procedure from Shi and Shum (2015), and details are given in section B of the supplemental appendix.

\section{Handling Covariates: Two-Step Estimation}

Here we describe a two-step estimation approach for this model, which resembles the two-step procedure in Iaryczower and Shum (2012b). This

${ }^{15}$ This is a weak assumption that is satisfied if each point in $\mathcal{B}_{0}$ either is in the interior of $\mathcal{B}_{E}$ or is a limit point of a sequence in the interior of $\mathcal{B}_{E}$. Unlike seemingly similar assumptions in the literature, it does not require the identified set $\mathcal{B}_{0}$ to have a nonempty interior. In this paper, numerical calculation of the identified sets for different values of $\vec{p}_{v}$ suggests that this assumption holds. 
is a simple and effective way to deal with a large number of covariates. Throughout, we let $X_{t}$ denote the set of covariates associated with case $t$, including the characteristics of the judges who are hearing case $t$.

In the first step, we estimate a flexible "reduced-form" model for the conditional probabilities $p_{v}(\vec{v} \mid X)$ of the vote outcomes given $X .{ }^{16}$ Specifically, we parameterize the probabilities of the eight feasible vote profiles using an eight-outcome multinomial logit model. ${ }^{17}$ Letting $i$ index the eight vote profiles, we have

$$
\begin{aligned}
& p_{v}\left(\vec{v}_{i} \mid X ; \beta\right)=\frac{\exp \left(X_{i}^{\prime} \beta_{i}\right)}{1+\sum_{i^{\prime}=1}^{7} \exp \left(X_{i^{\prime}} \beta_{i^{\prime}}\right)}, \quad i=1, \ldots, 7 ; \\
& p_{v}\left(\vec{v}_{8} \mid X ; \beta\right)=\frac{1}{1+\sum_{i^{\prime}=1}^{7} \exp \left(X_{i} \beta_{i^{\prime}}\right)},
\end{aligned}
$$

where $\vec{v}_{1}, \ldots, \vec{v}_{7}$ are the seven elements in $\mathcal{V}$ and $\vec{v}_{8}=011 .{ }^{18}$ Because the labeling of the three judges is arbitrary, it makes sense to impose an $e x$ changeability requirement on our model of vote probabilities. In particular, the conditional probability of a vote profile $\left(v_{1}, v_{2}, v_{3}\right)$ given case characteristics $X$ and judge covariates $\left(Z_{1}, Z_{2}, Z_{3}\right)$ should be invariant to permutations of the ordering of the three judges; that is, the vote probability $P\left(v_{1}, v_{2}, v_{3} \mid X, Z_{1}, Z_{2}, Z_{3}\right)$ should be exchangeable in $\left(v_{1}, Z_{1}\right),\left(v_{2}, Z_{2}\right)$, and $\left(v_{3}, Z_{3}\right)$ for all $X$. These exchangeability conditions imply restrictions on the coefficients on $\left(X, Z_{1}, Z_{2}, Z_{3}\right)$ in the logit choice probabilities, which greatly reduce the dimension of the unknown parameter. ${ }^{19}$

${ }^{16}$ This approach is commonplace in recent empirical applications of auction and dynamic game models (see, e.g., Cantillon and Pesendorfer 2006; Ryan 2012).

${ }_{17}$ The logit specification is convenient because it allows us to easily incorporate the exchangeability restrictions, as discussed below; also, it is capable of generating any conditional probability distribution of the discrete outcomes and thus is not restrictive. Finally, since we are using the multinomial logit model simply as a reduced-form description of the conditional probabilities of the vote outcomes, and not as a structural model of an agent's choice problem, the "red bus/blue bus" critique does not apply.

${ }^{18}$ By using a parameterization of the conditional vote probabilities $P(\vec{v} \mid X)$ that is continuous in $X$, we are also implicitly assuming that the equilibrium selection process is also continuous in $X$. Note that such an assumption is not needed if we estimate $P(\vec{v} \mid X)$ nonparametrically and impose no smoothness of these probabilities in $X$.

${ }^{19}$ In particular, symmetry implies the following constraints: (i) $\beta_{1,111}=\beta_{2,111}=\beta_{3,111}$, (ii) $\beta_{1,011}=\beta_{2,101}=\beta_{3,110}$, (iii) $\beta_{1,100}=\beta_{2,010}=\beta_{3,001}$, (iv) $\beta_{2,011}=\beta_{3,011}=\beta_{1,101}=\beta_{3,101}=$ $\beta_{1,110}=\beta_{2,110}$, (v) $\beta_{2,100}=\beta_{3,100}=\beta_{1,010}=\beta_{3,010}=\beta_{1,001}=\beta_{2,001}$, (vi) $\gamma_{011}=\gamma_{110}=\gamma_{101}$, and (vii) $\gamma_{001}=\gamma_{100}=\gamma_{010}$, where $\beta_{j, \vec{v}}$ is the coefficient on judge $j$ 's characteristics in the multinomial logit equation for the vote profile $\vec{v}$, and $\gamma_{\vec{v}}$ is the coefficient on the case characteristics in the multinomial logit equation for the vote profile $\vec{v}$. See also Menzel (2011) for a related discussion about the importance of exchangeability restrictions in Bayesian inference of partially identified models. 
Given the first-stage parameter estimates $\hat{\beta}=\left(\hat{\beta}_{1}, \ldots, \hat{\beta}_{7}\right)^{\prime}$, we obtain estimated vote probabilities $\hat{p}=\left(p\left(\vec{v}_{1} \mid X ; \hat{\beta}\right), \ldots, p\left(\vec{v}_{7} \mid X ; \hat{\beta}\right)\right)^{\prime}$. In the second stage, we use the estimated voting probability vector $\hat{p}$ to estimate the identified set of the model parameters $(\theta, \vec{\pi})$ using arguments from the previous section. This estimation procedure allows the underlying model parameters $(\theta, \vec{\pi})$ and the voting assignment $\mu$ to depend flexibly on $X .^{20}$

\section{Results}

\section{A. First-Stage Estimates}

The results from the first-stage estimation are presented in table 5. Since these are "reduced-form" vote probabilities, these coefficients should not be interpreted in any causal manner, but rather summarizing the correlation patterns in the data. Nevertheless, some interesting patterns emerge.

First, vote outcomes differ significantly depending on the type of crime considered in each case (cases involving aggravated assault, white-collar crimes, and theft are significantly less likely to be overturned in a divided decision than other cases) and in response to differences in legal issues (cases involving problems with jury instruction or sentencing in the lower courts are, on average, less likely to be overturned in a divided decision, while cases involving issues of sufficiency and admissibility of evidence are less likely to be overturned in unanimous decisions).

Vote outcomes also change with the partisan composition of the court. A Republican judge is less likely to be in the majority of a divided decision to overturn (less so in assault and white-collar cases) and more likely to be in the majority of a divided decision to uphold the decision of the lower court. At the same time, cases considered by courts composed of a majority of Republican judges, on average, have a significantly higher probability of being overturned in both unanimous and divided decisions. The first result indicates that this is due to the voting behavior of the Democrat judge when facing a Republican majority.

Finally, vote outcomes also differ on the basis of judges' judicial and political experience. Judges with more judicial and political experience, or with more years of experience in the court, are less likely to be in the majority of a divided decision to overturn. Having neither a female judge

\footnotetext{
${ }^{20}$ Both the estimation and the inference procedure described in the previous section can be used for each fixed value of $X=x$ in exactly the same way, only with $\hat{p}_{v}(\vec{v}), \vec{p}_{v}$, $p_{v}(\vec{v})$, and $\vec{p}_{v}$ replaced by $p_{v}(\vec{v} \mid x, \hat{\beta}), \vec{p}_{v}(x, \hat{\beta}), p_{v}(\vec{v} \mid x, \beta)$, and $\vec{p}_{v}(x, \beta) ;(\theta, \vec{\pi}, \mu)$ replaced by $(\theta(x), \vec{\pi}(x), \mu(\cdot \mid \cdot ; x))$; and $\hat{\Sigma}_{n}$ replaced by $\hat{\Sigma}_{n}(x)=\left[\partial \vec{p}_{v}(x, \hat{\beta}) / \partial \beta^{\prime}\right] \hat{\Sigma}_{\beta}\left[\partial \vec{p}_{v}(x, \hat{\beta}) / \partial \beta\right]$, where $\hat{\Sigma}_{\beta}$ is a consistent estimator of $\Sigma_{\beta}$ the asymptotic variance of $\sqrt{n}(\hat{\beta}-\beta)$, which can be obtained from the first stage. The consistency and the coverage probability theory go through in the logit case described above as long as $\Sigma_{\beta}$ is invertible.
} 
TABLE 5

First-Stage Estimates from a Multinomial Logit Model:

Baseline Vote Profile $(0,0,0)$

\begin{tabular}{|c|c|c|c|c|c|c|}
\hline & \multicolumn{6}{|c|}{$v=(v(i), v(k), v(m))$} \\
\hline & \multicolumn{2}{|c|}{$v=(1,1,1)$} & \multicolumn{2}{|c|}{$v=(1,0,1)$} & \multicolumn{2}{|c|}{$v=(0,1,0)$} \\
\hline & & Standard & & Standard & & Standard \\
\hline & Coefficient & Error & Coefficient & Error & Coefficient & Error \\
\hline \multicolumn{7}{|l|}{ Case-specific: } \\
\hline FedLaw & -.160 & .131 & -1.008 & .235 & -.296 & .271 \\
\hline Aggravated & -.217 & .271 & -1.254 & .520 & .585 & .553 \\
\hline White-Collar & -.406 & .231 & -.751 & .438 & .429 & .509 \\
\hline Theft & .042 & .241 & -1.362 & .563 & 1.432 & .505 \\
\hline Narcotics & -.260 & .243 & -.578 & .474 & .061 & .592 \\
\hline Republican Majority & .332 & .178 & 1.308 & .445 & -.549 & .376 \\
\hline Female & .050 & .165 & .212 & .346 & .136 & .326 \\
\hline \multicolumn{7}{|l|}{ Harvard-Yale } \\
\hline Majority & -.120 & .118 & -.263 & .277 & -.149 & .245 \\
\hline Jury Instruction & -.147 & .119 & -.913 & .359 & .227 & .216 \\
\hline Sentencing & -.341 & .130 & -.922 & .384 & -.081 & .266 \\
\hline Admissibility & -.333 & .099 & -.316 & .229 & .374 & .189 \\
\hline Sufficiency & -.543 & .115 & -.426 & .276 & -.268 & .226 \\
\hline \multicolumn{7}{|l|}{ Judge-specific: } \\
\hline$J(i)$ Republican & -.192 & .117 & -1.950 & .347 & .634 & .325 \\
\hline $\begin{array}{l}J(i) \text { Years of } \\
\text { Experience }\end{array}$ & -.003 & .004 & -.028 & .010 & .004 & .009 \\
\hline$J(i)$ Prior Judicial & & & & & & \\
\hline $\begin{array}{l}\text { Experience } \\
\text { (i) Prior Political }\end{array}$ & -.001 & .004 & -.046 & .012 & -.006 & .010 \\
\hline $\begin{array}{l}\text { Experience } \\
J(i) \text { Republican } \times\end{array}$ & .006 & .007 & -.041 & .022 & .034 & .015 \\
\hline $\begin{array}{l}\text { Assault } \\
\text { (i) Republican } \times\end{array}$ & -.021 & .162 & .918 & .464 & .037 & .394 \\
\hline $\begin{array}{l}\text { White-Collar } \\
\text { Ji) Republican } \times\end{array}$ & .110 & .137 & .861 & .447 & -.203 & .370 \\
\hline $\begin{array}{l}\text { Theft } \\
\text { J(i) Republican } \times\end{array}$ & -.175 & .157 & -.077 & .577 & -.910 & .447 \\
\hline Narcotics & -.075 & .141 & .416 & .483 & -.089 & .426 \\
\hline$J(k)$ Republican & -.192 & .117 & -.967 & .363 & -.324 & .430 \\
\hline $\begin{array}{l}J(k) \text { Years of } \\
\text { Experience } \\
J(k) \text { Prior Judicial }\end{array}$ & -.003 & .004 & -.019 & .014 & .015 & .012 \\
\hline $\begin{array}{l}J(k) \text { Prior Judicial } \\
\text { Experience } \\
J(k) \text { Prior Political }\end{array}$ & -.001 & .004 & -.004 & .014 & -.029 & .015 \\
\hline $\begin{array}{l}\text { Experience } \\
J(k) \text { Republican } \times\end{array}$ & .005 & .007 & -.047 & .033 & -.035 & .029 \\
\hline $\begin{array}{l}\text { Assault } \\
J(k) \text { Republican } \times\end{array}$ & -.021 & .162 & .729 & .611 & .028 & .575 \\
\hline $\begin{array}{l}\text { White-Collar } \\
J(k) \text { Republican } \times\end{array}$ & .110 & .137 & .483 & .582 & .558 & .536 \\
\hline $\begin{array}{l}\text { Theft } \\
J(k) \text { Republic } \times\end{array}$ & -.175 & .157 & 1.744 & .694 & -1.486 & .844 \\
\hline Narcotics & -.075 & .141 & .428 & .618 & -.325 & .630 \\
\hline $\begin{array}{l}J(m) \text { Republican } \\
J(m) \text { Years of }\end{array}$ & -.192 & .117 & -1.950 & .347 & .634 & .325 \\
\hline Experience & -.003 & .004 & -.028 & .010 & .004 & .009 \\
\hline
\end{tabular}

This content downloaded from 131.215.071.079 on April 18, 2018 09:08:51 AM

All use subject to University of Chicago Press Terms and Conditions (http://www.journals.uchicago.edu/t-and-c). 
TABLE 5 (Continued)

\begin{tabular}{|c|c|c|c|c|c|c|}
\hline & \multicolumn{6}{|c|}{$v=(v(i), v(k), v(m))$} \\
\hline & \multicolumn{2}{|c|}{$v=(1,1,1)$} & \multicolumn{2}{|c|}{$v=(1,0,1)$} & \multicolumn{2}{|c|}{$v=(0,1,0)$} \\
\hline & & Standard & & Standard & & Standard \\
\hline & Coefficient & Error & Coefficient & Error & Coefficient & Error \\
\hline \multicolumn{7}{|l|}{$J(m)$ Prior Judicial } \\
\hline Experience & -.001 & .004 & -.046 & .012 & -.007 & .009 \\
\hline$J(m)$ Prior Political & & & & & & \\
\hline Experience & .005 & .007 & -.042 & .022 & .034 & .015 \\
\hline$J(m)$ Republican $\times$ & & & & & & \\
\hline Assault & -.021 & .162 & .918 & .464 & .037 & .394 \\
\hline$J(m)$ Republican $\times$ & & & & & & \\
\hline White-Collar & .110 & .137 & .861 & .447 & -.203 & .370 \\
\hline$J(m)$ Republican $\times$ & & & & & & \\
\hline Theft & -.175 & .157 & -.077 & .577 & -.910 & .447 \\
\hline$J(m)$ Republican $\times$ & & & & & & \\
\hline Narcotics & -.075 & .141 & .416 & .483 & -.089 & .426 \\
\hline Constant & -.394 & .215 & (dropped) & & -4.455 & .517 \\
\hline
\end{tabular}

on the panel nor a majority of graduates from Harvard or Yale Law Schools (a possible club effect) is significantly related to vote outcomes.

\section{B. Second-Stage Estimates: Preferences and Information}

In the second stage we use the estimated voting probability vector $\hat{p}=$ $p(\vec{v} \mid X ; \hat{\beta})$ to estimate the identified set of the model parameters $(\theta, \vec{\pi})$. To present the results, we fix benchmark case and judge characteristics and later on introduce comparative statics from this benchmark. For our benchmark case we consider a white-collar crime prosecuted under federal law, in which the major legal issue for appeal is admissibility of evidence. Judges 1 and 2 are Republican, and judge 3 is a Democrat (so that the majority of the court is Republican). All three judges are male, and at most one of the judges has a law degree from Harvard or Yale. The three benchmark judges differ in their years of court experience, as well as prior judicial and political experience. (See table A.3 in the supplemental appendix for the full benchmark specification.)

The left panel of figure 2 plots points in the EIS for an agnostic prior belief, $\rho=0.5$, which we take as a benchmark to present our results. For simplicity, we begin by presenting results for a symmetric model, in which $\pi_{i}=\pi$ for all $i \in N$.

Two features of the EIS are immediately apparent from the figure. First, as in Section IV.A, the range of values of the bias parameter $\pi$ that are consistent with the data for a given value of competence is increasing in $q$. Thus, high-ability judges can be highly predisposed to uphold or to overturn, but low-ability judges must be relatively malleable, willing to overturn (uphold) even when it is slightly more (less) likely than not that 
the trial court's decision is incorrect $(\pi \approx 1 / 2)$. Second, because the distribution of vote profiles in the data is asymmetric in favor of upholding the decision of the lower court, the EIS for $\rho=0.5$ is asymmetric toward larger values of $\pi$, indicating a higher information hurdle to overturn the decision of the lower court. Thus, with an uninformative prior, malleable judges of all competence levels are consistent with the data, but judges who are highly predisposed to uphold can be consistent with the data only if they are highly competent, and judges that are highly predisposed to overturn are not consistent with the data (irrespective of their competence level).

For comparison purposes, the right panel of figure 2 plots the EIS for a value of $\rho$ that approximates the empirical frequency of cases in which the court overturned the decision of the lower courts, $\rho=0.2$. In this case the prior belief that the trial's court decision is flawed is relatively low. Thus, when private signals are not too informative, only judges who are predisposed to overturn $(\pi<1 / 2)$ can vote in a way consistent with the data. To understand this inverse relationship between $\rho$ and $\pi_{i}$ among points in the EIS, recall that judge $i$ votes to overturn given information $\mathcal{I}$ if and only if $\operatorname{Pr}^{i}(\omega=1 \mid \mathcal{I}) \geq \pi_{i}$, which can be written in terms of the relative likelihood of the event $\mathcal{I}$ in states $\omega=1$ and $\omega=0$ as

$$
\frac{\operatorname{Pr}^{i}(\mathcal{I} \mid \omega=1)}{\operatorname{Pr}^{i}(\mathcal{I} \mid \omega=0)} \geq \frac{\pi_{i}}{1-\pi_{i}} \frac{1-\rho}{\rho} .
$$

The results for the EIS with heterogeneous preferences extend naturally the results of figure 2 for the symmetric model: while low-competence judges must be homogeneous and relatively malleable (willing to uphold or overturn with little supporting information) in order to be consistent with the data, competent judges can have highly heterogeneous preferences and still generate a distribution of vote profiles consistent with the data. To illustrate this result in a simple plot, we introduce a measure of preference heterogeneity:

$$
H(\vec{\pi})=\sum_{i \in N} \sum_{j \neq i}\left(\pi_{i}-\pi_{j}\right)^{2}
$$

Our index of preference heterogeneity increases as judges' bias parameters are farther apart from one another, reaching a theoretical maximum of two, and decreases as judges' preferences are closer to each other's, reaching a minimum of zero when all judges have the same preferences.

Figure 3 plots pairs of quality of information and preference heterogeneity that are consistent with points $(\vec{\pi}, q)$ in the EIS for $\rho=0.5$, for the asymmetric model in which judges' preferences $\pi_{i}, i=1,2,3$, are not restricted to be identical. For low quality, only very homogeneous courts $(H \rightarrow 0)$ are consistent with the data; but as competence increases the 

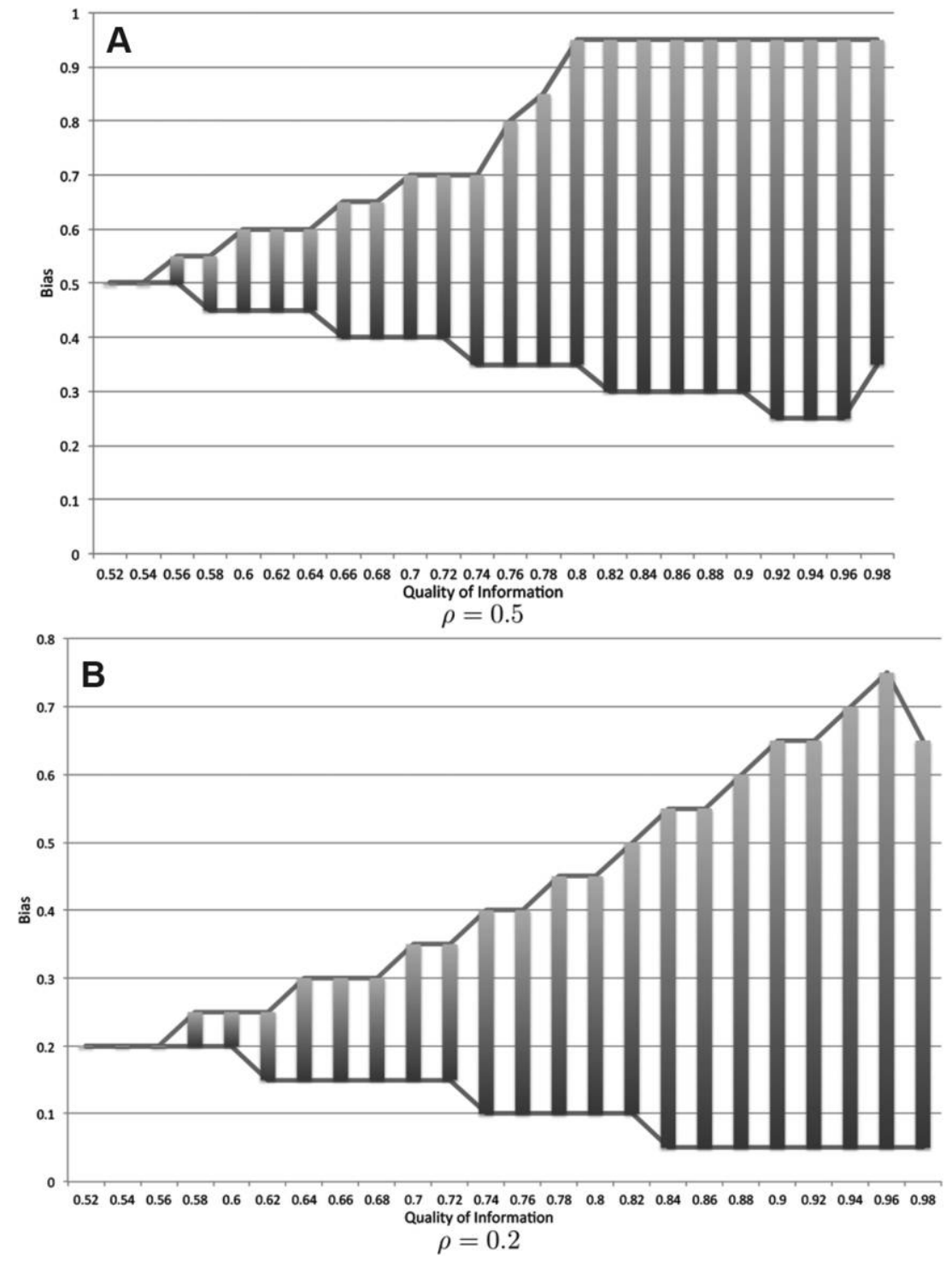

FIG. 2.- The figure plots points $(\pi, q)$ in the EIS for $\rho=0.5$ (panel A) and $\rho=0.2$ (panel B) in a symmetric model, where $\pi_{i}=\pi$ for all $i \in N$. Color version available as an online enhancement.

allowed heterogeneity in preferences increases as well, reaching values close to one for high levels of $q$.

This result is interesting in its own right because it clarifies that high unanimity rates (a feature of our voting data) do not imply common in- 


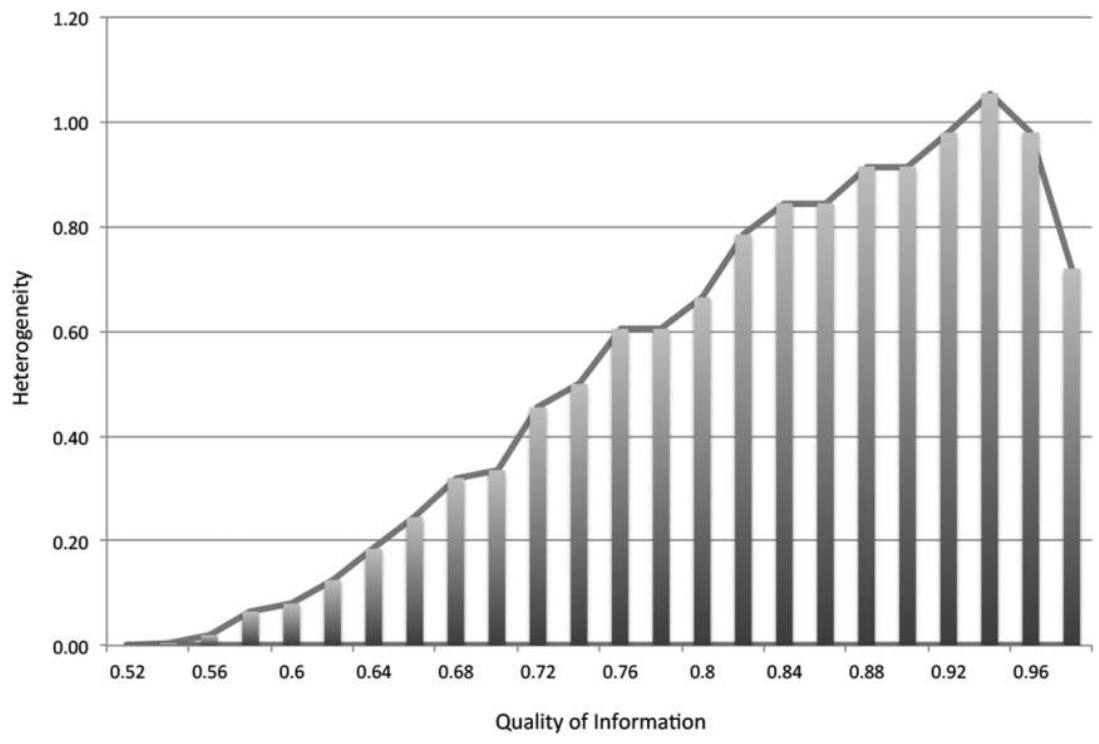

FIG. 3.-Pairs $(H, q)$ consistent with points $(\vec{\pi}, q)$ in the EIS for $\rho=0.5$, asymmetric model. Color version available as an online enhancement.

terests at an ex ante stage. Thus, neither preference homogeneity nor external motives, such as an intrinsic desire to compromise or to put forward a "unified" stance in each case, are required to rationalize the data. While low-quality judges would agree as much as they do in the data only if they had very similar preferences, deliberation among competent judges can generate the high frequency of unanimous votes observed in the data without requiring these auxiliary motives.

Comparative statics. - In the discussion above, we have focused on the benchmark case and court characteristics. It should be clear, however, that both the identified set and the set of equilibrium outcomes for each point in the identified set are functions of the observable characteristics that enter the first-stage multinomial logit model. Thus, proceeding as above, we can quantify the changes in types and outcomes associated with alternative configurations of the cases and/or the courts under consideration. To illustrate this, we evaluate the effect of changing the nature of the crime considered in the case from a white-collar crime to theft on judges' preferences: are justices more or less predisposed to overturn the lower court in theft cases?

The results are illustrated in figure 4 . The figures show points in whitecollar EIS not in the theft EIS (blue) and points in the theft EIS not in the white-collar EIS (red). The figures suggest that changing from whitecollar to theft crimes makes the average judge less prone to overturning the lower court (left panel) and reduces the level of disagreement in the 

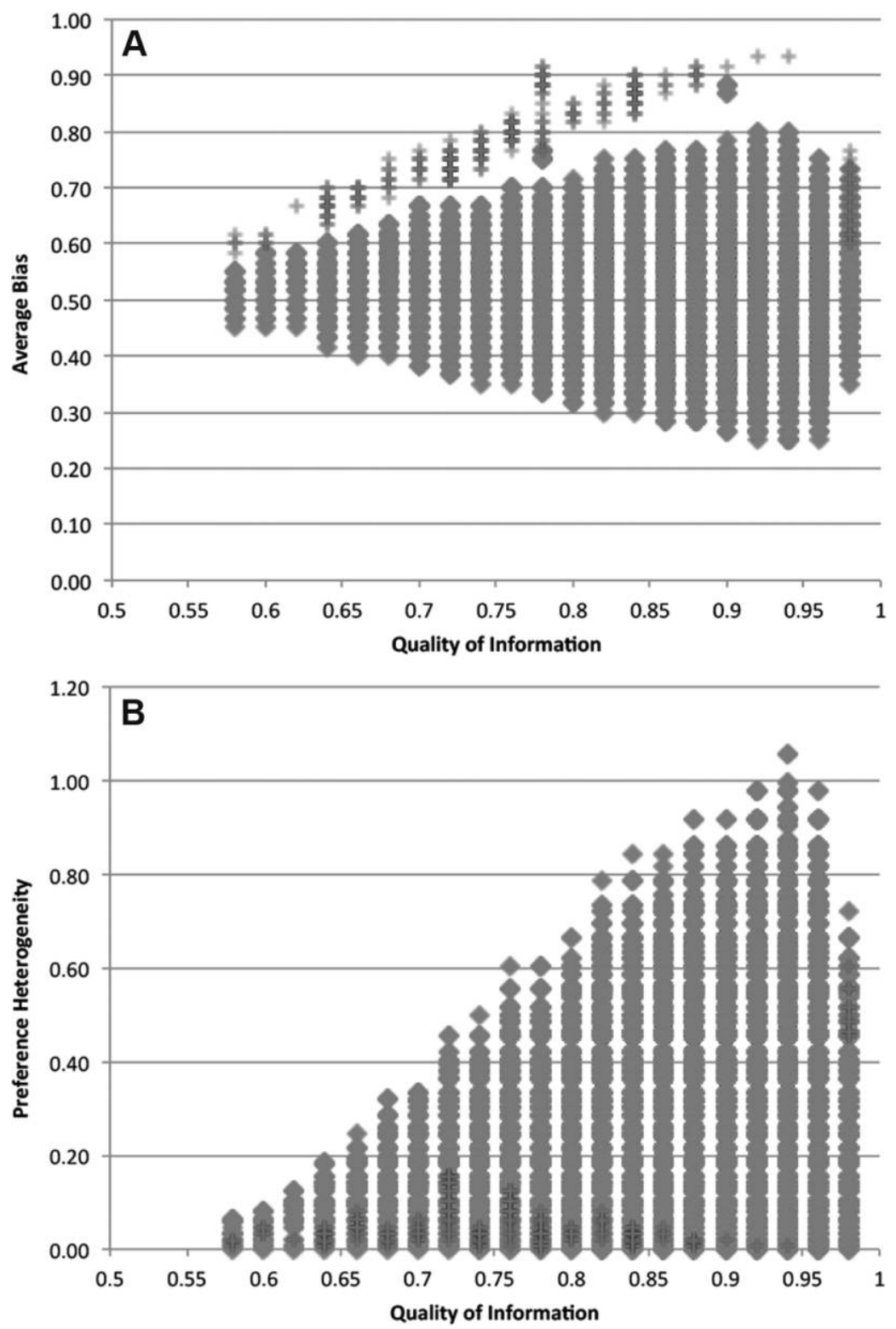

FIG. 4.-Points in white-collar EIS not in the theft EIS (blue diamonds), and points in the theft EIS not in the white-collar EIS (red crosses). The $y$-axis plots the average bias (panel A) and preference heterogeneity (panel B). The $x$-axis plots quality of information $q$. Color version available as an online enhancement. 
court (right panel). The theft EIS excludes the more heterogeneous courts and the courts with judges biased more toward upholding in the white-collar EIS.

\section{Equilibrium Outcomes with Deliberation}

In the previous section we described the set of characteristics of members of the court that are consistent with the data (points $(\theta, \vec{\pi})$ in the EIS). We now use these results to evaluate the set of outcomes that are consistent with the data. We know the voting probabilities since we used them to estimate the EIS in the first place. But knowing the set of parameters consistent with the data allows us to compute more interesting measures of payoff-relevant outcomes. In particular, we focus on the probability that the court reaches an incorrect decision after deliberating and voting strategically.

Note that for any given point $(\theta, \vec{\pi}) \in \mathcal{A}_{0}$ and any communication equilibrium $\mu \in M(\theta, \vec{\pi})$, we can compute the probability that the court reaches an incorrect decision, $\varepsilon(\mu,(\theta, \vec{\pi}))$. This probability of error $\varepsilon(\mu,(\theta, \vec{\pi}))$ is the weighted average of the type I error (overturn when should uphold),

$$
\varepsilon_{\mathrm{I}}(\mu,(\theta, \vec{\pi}))=\operatorname{Pr}(v=1 \mid \omega=0)=\sum_{\vec{t}} \sum_{\vec{v}: v=1} \mu(\vec{v} \mid \vec{t}) p(\vec{t} \mid \omega=0),
$$

and the type II error (uphold when should overturn)

$$
\varepsilon_{\mathrm{II}}(\mu,(\theta, \vec{\pi}))=\operatorname{Pr}(v=0 \mid \omega=1)=\sum_{\vec{t}} \sum_{\vec{v}: v=0} \mu(\vec{v} \mid \vec{t}) p(\vec{t} \mid \omega=1),
$$

that is, ${ }^{21}$

$$
\varepsilon(\mu,(\theta, \vec{\pi}))=(1-\rho) \varepsilon_{\mathrm{I}}(\mu,(\theta, \vec{\pi}))+\rho \varepsilon_{\mathrm{II}}(\mu,(\theta, \vec{\pi})) .
$$

For each point $(\theta, \vec{\pi}) \in \mathcal{A}_{0}$ there are in fact multiple equilibria $\mu \in$ $M(\theta, \vec{\pi})$, each being associated with a certain probability of error $\varepsilon(\mu,(\theta, \vec{\pi}))$ computed as in (15). Thus, for each point in the EIS, there is a set of error probabilities that can be attained in equilibrium. In order to describe the range of possible equilibrium outcomes for court configurations consistent with the data, we focus on the maximum and minimum equilibrium probability of error for each point in the EIS.

There are two possible sets of such bounds that a researcher might find valuable, depending on the question at hand. First, we can compute the maximum and minimum error probabilities across equilibria that

\footnotetext{
${ }^{21}$ Note that both the type I error and the type II error are functions of the model parameters $\mu, \theta, \vec{\pi}$, and inference on them amounts to projecting the EIS of the model parameters onto the range of these functions.
} 
are consistent with the observed data, $\vec{p}_{v}, \bar{\varepsilon}^{*}\left(\theta, \vec{\pi}, p_{v}\right)$, and $\underline{\varepsilon}^{*}\left(\theta, \vec{\pi}, p_{v}\right)$. These bounds rule out error probabilities that either are not attainable in equilibrium given the parameters $(\theta, \vec{\pi})$ or are attainable by mixtures of equilibria that would lead to a distribution over vote profiles that differs from the one observed in the data. Formally, for each point $(\theta, \vec{\pi})$ in the EIS and data $\vec{p}_{v}$, we define

$$
\bar{\varepsilon}^{*}\left(\theta, \vec{\pi}, p_{v}\right) \equiv \max _{\mu \in M(\theta, \vec{\pi})}\left\{\varepsilon(\mu,(\theta, \vec{\pi})) \text { s.t. } p_{v}(\vec{v})=\sum_{\vec{t}} \mu(\vec{v} \mid \vec{t}) p(\vec{t} ; \theta)\right\},
$$

and similarly for $\underline{\varepsilon}^{*}\left(\theta, \vec{\pi}, p_{v}\right) .^{22}$

A second, more expansive, criterion is to consider the maximum and minimum probability of error across all equilibria:

$$
\begin{aligned}
& \bar{\varepsilon}(\theta, \vec{\pi}) \equiv \max _{\mu \in M(\theta, \vec{\pi})} \varepsilon(\mu,(\theta, \vec{\pi})), \\
& \underline{\varepsilon}(\theta, \vec{\pi}) \equiv \min _{\mu \in M(\theta, \vec{\pi})} \varepsilon(\mu,(\theta, \vec{\pi})) .
\end{aligned}
$$

Unlike expression (16), expression (17) includes error probabilities that are attainable through equilibria that are not consistent with the observed data. The logic behind (17) is that equilibrium selection in a given sample is not informative about equilibrium selection in a counterfactual (or in a different sample). Thus, although in the particular data at hand we can rule out that these equilibria were played for these parameter values, it is conceivable that these outcomes can be produced if judges were to play a different selection of equilibria in a counterfactual.

Figure 5 plots the minimum and maximum probability of error in equilibria consistent with the data for pairs of preference heterogeneity and competence $(H, q)$ consistent with points in the EIS for $\rho=0.5 .^{23}$

Consider first the minimum error probability, on the left panel. For low competence, $q$, only very homogeneous courts, composed entirely of malleable judges, are consistent with the data. These courts are highly inaccurate, even after pooling information, and correspondingly make wrong decisions very often (about 45 percent of the time as $q \rightarrow 1 / 2$ ). As ability increases, however, more heterogeneous courts can also be consistent with the data. These more able courts are capable of producing decisions with a much lower error rate, even when they are quite heterogeneous.

The right panel focuses on the maximum probability of error in equilibria consistent with the data. The difference between the best and worst

\footnotetext{
${ }^{22}$ Note that because $M(\theta, \vec{\pi})$ is a convex set and the constraint $p_{v}(\vec{v})=\Sigma_{\vec{t}} \mu(\vec{v} \mid \vec{t}) p(\vec{t} ; \theta)$ is linear in $\mu, \mu$ can be replaced with a linear combination of elements in $M(\theta, \vec{\pi})$ without affecting the value of $\bar{\varepsilon}^{*}\left(\theta, \vec{\pi}, p_{v}\right)$ or $\underline{\varepsilon}^{*}\left(\theta, \vec{\pi}, p_{v}\right)$. Therefore, when considering equilibria consistent with the data, we are not assuming that the same equilibrium is played in every case.

${ }^{23}$ When there are multiple points $(\vec{\pi}, q)$ such that $H(\vec{\pi})=H$, the figure plots the average of extrema across these points.
} 

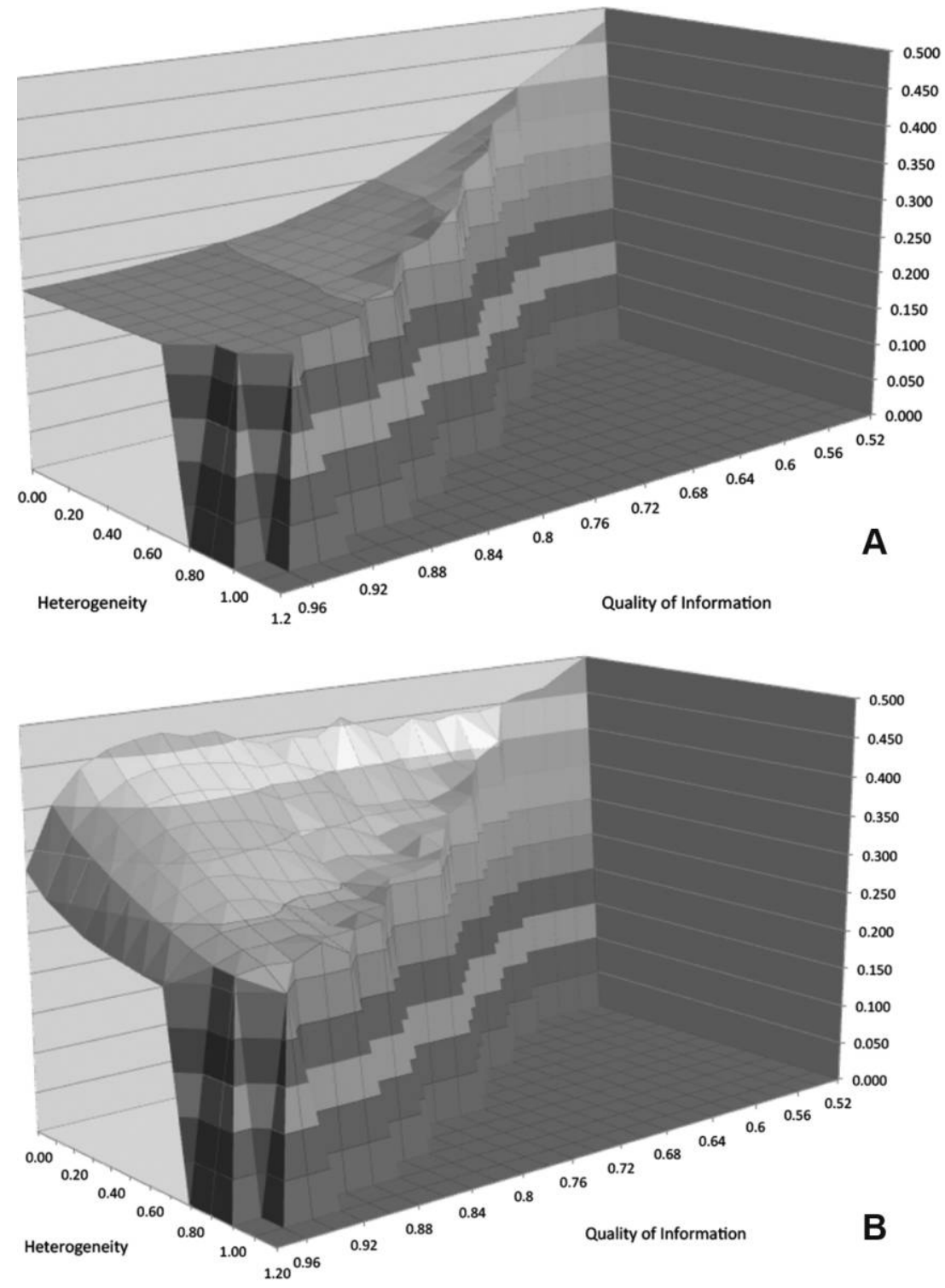

FIG. 5.-Minimum (panel A) and maximum (panel B) probability of error in equilibria consistent with the data, for pairs of preference heterogeneity and competence $(H, q)$ consistent with points in the EIS for $\rho=0.5$ (average of extrema across points $(\vec{\pi}, q)$ such that $H(\vec{\pi})=H)$. Color version available as an online enhancement.

This content downloaded from 131.215.071.079 on April 18, 2018 09:08:51 AM

All use subject to University of Chicago Press Terms and Conditions (http://www.journals.uchicago.edu/t-and-c). 
equilibria is small for homogeneous courts of low competence and heterogeneous courts with high competence but is relatively large for courts composed of competent judges with aligned preferences. The reason is that errors in the worst equilibrium remain high as ability increases precisely when courts are homogeneous. In fact, the last column of table 4 shows that the example in Section III generates a vote distribution equal to the one observed in the data. On the other hand, the maximum equilibrium probability of error decreases sharply with the heterogeneity of the court when courts are competent. Thus, heterogeneous courts can be rationalized as generating the observed voting data, but only if they are competent and play equilibria in which they use their information effectively.

The key consideration we should keep in mind here is that this is not a theoretical result, but a combination of data and equilibrium restrictions. Heterogeneous courts have to be "better"-in the sense that judges must have more precise information and, for any given level of quality, must shed the worse equilibria - in order to be consistent with the data. The reason why very heterogeneous courts must be sufficiently effective to be consistent with the data, on the other hand, comes directly from the equilibrium conditions in the voting stage and the feature of our observed data.

To see how, consider without loss of generality judges with bias parameters $\pi_{1}, \pi_{2}$, and $\pi_{3}$ such that $\pi_{1}<\pi_{2}=\pi_{3}$. As the heterogeneity in the bias parameters increases, the court is more and more predisposed to vote $\vec{v}=\{100\}$. However, in our observed data (refer to the last column of table 4$), \vec{v}=\{100\}$ is not predominately more likely than $\vec{v}=\{010\}$ and $\vec{v}=\{001\}$. Thus, in order to be consistent with this feature of the data, the judges need to be induced to play $\{010\}$ and $\{001\}$ with nonnegligible probability. Take $\{001\}$ as an example. Given a signal profile $\vec{t}$, in order for $\{001\}$ to be the equilibrium outcome, we need judge 1 to vote 0 given $t_{1}$, judge 2 to vote 0 given $t_{2}$, and judge 3 to vote 1 given $t_{3}$. But note that in equilibrium judge $i$ votes to overturn given signal $t_{i}$ if and only if

$$
\operatorname{Pr}\left(\omega=1 \mid v_{i}=1, t_{i}, \operatorname{Piv}^{i} ;(\vec{q}, \rho, \mu)\right) \geq \pi_{i}
$$

and votes to uphold given signal $t_{i}$ if and only if

$$
\operatorname{Pr}\left(\omega=1 \mid v_{i}=0, t_{i}, \operatorname{Piv}^{i} ;(\vec{q}, \rho, \mu)\right) \leq \pi_{i} .
$$

To achieve this when heterogeneity in the judges' bias parameters $\pi_{i}$ is very high, the inference of judge 3 about her fellow panel members' information must be powerful enough to sway her away from her initial predisposition. This cannot happen when she knows that the other judges' information is useless or if the strategy profile is not sufficiently informative.

This logic also shows why courts composed of competent judges with similar preferences can produce bad outcomes. Homogeneous courts put 
less demanding constraints on equilibrium beliefs and thus on equilibrium behavior $\mu$. Put informally, with less diversity of preferences there are fewer checks on equilibrium group behavior. Moreover, the possibility of being able to sustain bad equilibria increases with the precision of judges' information. This is the same reason why judges' ability must be relatively high if they are to be consistent with the data when courts are heterogeneous: it is precisely when individuals believe that the fellow panel members have precise information that equilibrium inferences become more powerful.

Figure 6 reproduces figure 5 across all equilibria. The right panel of figure 6 plots the mapping of points in the EIS to the probability of error. As the figure illustrates, $\bar{\varepsilon}(\cdot)$ is qualitatively similar to the maximum probability of error across equilibria consistent with the data $\bar{\varepsilon}^{*}\left(\cdot, p_{v}\right)$.

The left panel plots the minimum equilibrium probability of error across all equilibria. As before, the rate of errors in the best equilibria decreases with competence; but now the minimum error probability approaches zero as $q \rightarrow 1$, even when judges are highly heterogeneous. This contrasts with the results for equilibria consistent with the data, in which the minimum equilibrium error probability was bounded above by 20 percent, even as $q \rightarrow 1$. The intuition for this result is straightforward. Note that in a large sample, given a prior $\rho=0.5$, an unbiased highquality court playing the best equilibrium would uphold roughly 50 percent of the time. Recall, however, that in the data, the court upholds more than 75 percent of the time. This means that a court can match the data only by making a relatively large number of errors.

The logic is further emphasized in figure A.2 in the supplemental appendix, which reproduces figures 5 and 6 for a prior of $\rho=0.2$. Since in this case the prior is close to the frequency with which the court overturns the trial courts in the data, the minimum and maximum probabilities of error in the equilibria consistent with the data are lower overall, and the probability of error in the best equilibrium consistent with the data goes to zero as $q$ goes to one. With this caveat, the mapping of court characteristics to equilibrium outcomes with $\rho=0.2$ is qualitatively similar to that for $\rho=0.5$.

\section{The Impact of Deliberation}

Having described the outcomes attained in equilibria with deliberation, our next goal is to quantify the effect of deliberation: how much do outcomes differ because of deliberation?

To do this, we compare equilibrium outcomes with deliberation with the outcomes that would have arisen in a counterfactual scenario in which judges are not able to talk with one another before voting. In particular, for each point $(\theta, \vec{\pi})$ in the EIS, we compare the maximum and 




FIG. 6.-Minimum (panel A) and maximum (panel B) probability of error in all equilibria, for pairs of preference heterogeneity and competence $(H, q)$ consistent with points in the EIS for $\rho=0.5$ (average of extrema across points $(\vec{\pi}, q)$ such that $H(\vec{\pi})=H$ ). Color version available as an online enhancement.

minimum error probabilities in equilibria with deliberation with the corresponding maximum and minimum error probabilities in responsive Bayesian Nash equilibria (BNE) of the voting game without communication, $\bar{\varepsilon}^{N D}(\theta, \vec{\pi})$ and $\underline{\varepsilon}^{N D}(\theta, \vec{\pi})$.

To carry out this comparison, we solve for all responsive BNE of the nondeliberation game, for all parameter points in the EIS. In the game without deliberation, the strategy of player $i$ is a mapping $\sigma_{i}:\{0,1\} \rightarrow[0,1]$, 
where $\sigma_{i}\left(t_{i}\right)$ denotes the probability of voting to overturn given signal $t_{i}$. A BNE is a strategy profile $\sigma$ such that each judge $i$ s strategy is a best response to the voting strategy of the other judges in the court. In particular, it is easy to show that $\sigma_{i}\left(t_{i}\right)>0(<1)$ only if $\operatorname{Pr}\left(\omega=1 \mid t_{i}, \operatorname{Piv}^{i}\right) \geq \pi_{i}\left(\leq \pi_{i}\right)$ or

$$
\frac{\operatorname{Pr}\left(t_{i} \mid \omega=1\right)}{\operatorname{Pr}\left(t_{i} \mid \omega=0\right)} \frac{\operatorname{Pr}\left(\operatorname{Piv}^{i} \mid \omega=1 ; \sigma\right)}{\operatorname{Pr}\left(\operatorname{Piv}^{i} \mid \omega=0 ; \sigma\right)} \geq \frac{\pi_{i}}{1-\pi_{i}} \frac{1-\rho}{\rho} .
$$

Following the convention in the literature, we say that a BNE equilibrium $\sigma$ is responsive if the probability that the court overturns the decision of the lower court is not invariant to judges' private information. More specifically, let $\operatorname{Pr}(v=1 \mid \vec{t} ; \sigma)$ denote the probability that the court overturns the decision of the lower court when judges receive signals $\vec{t}$ and vote according to $\sigma$. Then a BNE $\sigma$ is responsive if there exist two signal realizations, $\vec{t}$ and $\vec{t}^{\prime}$, such that $\operatorname{Pr}(v=1 \mid \vec{t} ; \sigma) \neq \operatorname{Pr}\left(v=1 \mid \vec{t}^{\prime} ; \sigma\right)$. Characterizing responsive equilibria in the nondeliberation game is straightforward but somewhat cumbersome, because the set of $\mathrm{BNE}$ is not convex and a number of different strategy profiles can form a BNE for different parameter values (all judges mix after a 1 signal and uphold after a 0 signal, two judges mix after a 1 signal and uphold after a 0 signal while the third overturns, etc.). We discuss this further in the supplemental appendix (sec. D).

We begin by contrasting the probability of error with and without deliberation for all comparable points in the EIS, that is, for points in the EIS in which there exists a responsive equilibrium of the game without deliberation. We focus first on how the effect of deliberation changes as a function of initial disagreement among judges in the panel.

Figure 7 plots the maximum and minimum equilibrium probabilities of error with and without deliberation for various levels of information precision $q$ and prior beliefs $\rho$. The bounds on equilibrium errors are plotted as a function of the degree of preference heterogeneity in the court, for levels of heterogeneity consistent with points in the EIS (values $H$ such that $H=H(\vec{\pi})$ for some $\left.(\vec{\pi}, \theta) \in \mathcal{A}_{0}\right)$. Each panel plots the maximum and minimum probabilities of error in (i) all equilibria with deliberation (black), (ii) in equilibria of the voting game with deliberation that are consistent with the data (dotted), and (iii) in responsive equilibria without deliberation (red). ${ }^{24}$

The results show that deliberation can be useful when the court is heterogeneous but will generally be either ineffectual (all equilibria) or

\footnotetext{
${ }^{24}$ Note that the maximum level of preference heterogeneity consistent with the data is increasing in $q$ (fig. 3). As a result, the solid black lines extend for a larger range of values of $H$ as we move from the figures in the bottom (for $q=0.70$ ) to the figures in the top (for $q=$ $0.90)$.
} 

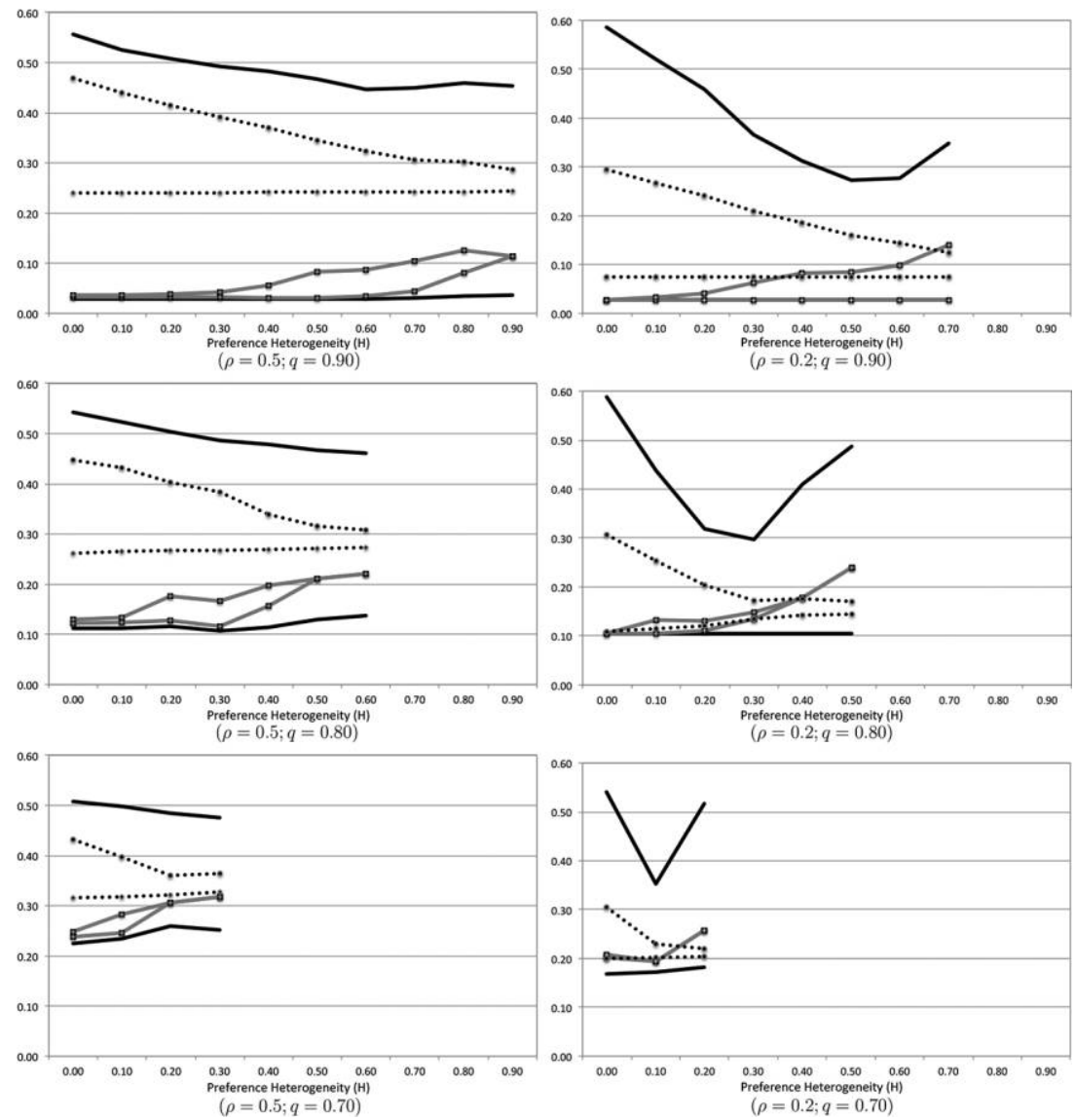

FIG. 7.-Probability of mistakes with and without deliberation for values of preference heterogeneity $H$ consistent with points $(\vec{\pi}, \theta)$ in the EIS for $\rho=0.5$ (left) and $\rho=0.2$ (right). The $y$-axis is the probability of error, and the $x$-axis is the degree of preference heterogeneity in the court. Minimum and maximum equilibrium probability of error in (i) all equilibria with deliberation (solid black), (ii) in equilibria with deliberation consistent with the data (dotted), and (iii) in responsive equilibria without deliberation (solid red, with marker). Color version available as an online enhancement.

counterproductive (e.g., consistent with the data) when the court is relatively homogeneous.

Consider first all equilibria with deliberation. Note that since there is always a "babbling" equilibrium, in which all messages are interpreted as uninformative, this set includes the set of equilibria without deliberation. Thus, $\underline{\varepsilon}(\theta, \vec{\pi}) \leq \underline{\varepsilon}^{N D}(\theta, \vec{\pi}) \leq \bar{\varepsilon}^{N D}(\theta, \vec{\pi}) \leq \bar{\varepsilon}(\theta, \vec{\pi})$ for all points $(\theta, \vec{\pi})$ in 
the EIS. Still, the comparison allows us to put an upper bound on the potential gain or loss that can be attributed to deliberation under any equilibrium selection rule. ${ }^{25}$ In fact, figure 7 shows that when the court is relatively homogeneous ( $H$ small $)$, the biggest possible improvement that can be attributed to deliberation is fairly small, under any possible equilibrium selection rule one could use. On the other hand, as the heterogeneity of preferences increases, mistakes in equilibria without deliberation become more frequent (both $\underline{\varepsilon}^{N D}(\theta, \vec{\pi})$ and $\bar{\varepsilon}^{N D}(\theta, \vec{\pi})$ shift up) while the probability of error in the best equilibrium with deliberation remains flat. This shows that at least for some initial prior beliefs, deliberation can have a nonnegligible positive effect on outcomes when the level of initial disagreement in the court is relatively high.

Considering equilibria consistent with the data allows a more straightforward assessment of the effect of deliberation. Because the set of outcomes of voting with deliberation in equilibria consistent with the data does not necessarily include the set of outcomes of voting without deliberation, nor is it ranked relatively to it in any way ex ante, the comparison with the counterfactual allows a more conclusive evaluation of the effect of deliberation.

The results reinforce our previous conclusions. As the figures show, in fact, for low levels of conflict, all responsive equilibria of the game without deliberation lead to a lower probability of mistakes than all equilibria consistent with the data of voting with deliberation. Thus, when courts are relatively homogeneous, prevote deliberation leads to a larger incidence of errors than responsive equilibria without deliberation, even when we consider the best possible equilibrium with deliberation and the worst responsive equilibrium without deliberation.

As before, this result changes when conflicts of interest among judges increase, as a result of two effects. First, as we have seen already, the probability of error in voting without deliberation increases with the heterogeneity of preferences in the court. In addition, the maximum probability of error of voting with deliberation in equilibria consistent with the data decreases with heterogeneity (recall fig. 5). For $\rho=0.5$, this implies that the negative effect of deliberation on outcomes diminishes with heterogeneity. But for $\rho=0.2$, where the errors in equilibria consistent with the data are lower to begin with, this means that deliberation actually im-

${ }^{25}$ Recall that our equilibrium concept in the game with deliberation is agnostic about the possible communication protocol judges might be using. Thus, a large gain/loss can be due to different equilibrium behavior for a fixed communication protocol or to our ignorance about which particular communication protocol judges could be using. On the other hand, we know that the equilibrium outcomes of any communication protocol judges could be using is contained in the set of outcomes of communication equilibria. Thus, the maximum gain/loss that can be attributed to deliberation provides an upper bound on the effect of deliberation. 
proves on no deliberation when the court is sufficiently heterogeneous. Overall, the results indicate that voting after deliberation can reduce errors when courts are sufficiently heterogeneous but leads to more erroneous decisions than what we would obtain in responsive equilibria without deliberation when courts are homogeneous.

Figure 8 presents the results from a different perspective. The panels in the figure reproduce the structure of figure 7 , but they do so for a fixed level of heterogeneity of preferences and prior beliefs, plotting errors as a function of the level of quality of information in the court. As the figures illustrate, deliberation tends to increase errors in the court when judges' private information is very precise: the probability of mistakes in all responsive equilibria without deliberation is fairly small and very close to the minimum probability of error across all equilibria with deliberation. Moreover, the probability of errors in all equilibria without deliberation is significantly lower than the probability of errors with prevote deliberation in any equilibrium consistent with the data. However, the relative efficacy of voting with deliberation increases as judges' private information gets less precise. In fact, for some values in the EIS for $\rho=0.2$, deliberation dominates no deliberation when judges' information is sufficiently imprecise. This result is intuitive: exchanging information before the vote can help precisely when it allows judges to overcome deficiencies in their own private information.

Figures 7 and 8 show how prevote deliberation can be beneficial when the court is heterogeneous or the quality of justices' private information is low. But just how typical is such a court configuration in comparable points in the EIS? Figure A.3 in the supplemental appendix plots the correspondence between the $\min / \max$ probability of error in responsive equilibria without deliberation and the $\min / \max$ probability of error in equilibria with deliberation, for both all equilibria and equilibria consistent with the data. The figure shows that while deliberation typically leads to higher error rates than what can be achieved in responsive equilibria without deliberation, it can also be beneficial for a range of points in the EIS, in particular when $\rho=0.2$. This reassures us that the picture presented in figures 7 and 8 is representative of the results across the EIS.

\section{E. Remarks and Robustness Checks}

We follow with a series of supplemental results and robustness checks.

Confidence set.- Up to this point, we have restricted the comparison between communication equilibria and responsive equilibria without deliberation to points in the estimated identified set. These are court types that are consistent with the point estimate of the vote probabilities, $\hat{p}(X)$. When we incorporate the uncertainty in our estimate of the true vote probability $p(X)$, the set of types that are consistent with the data is given 

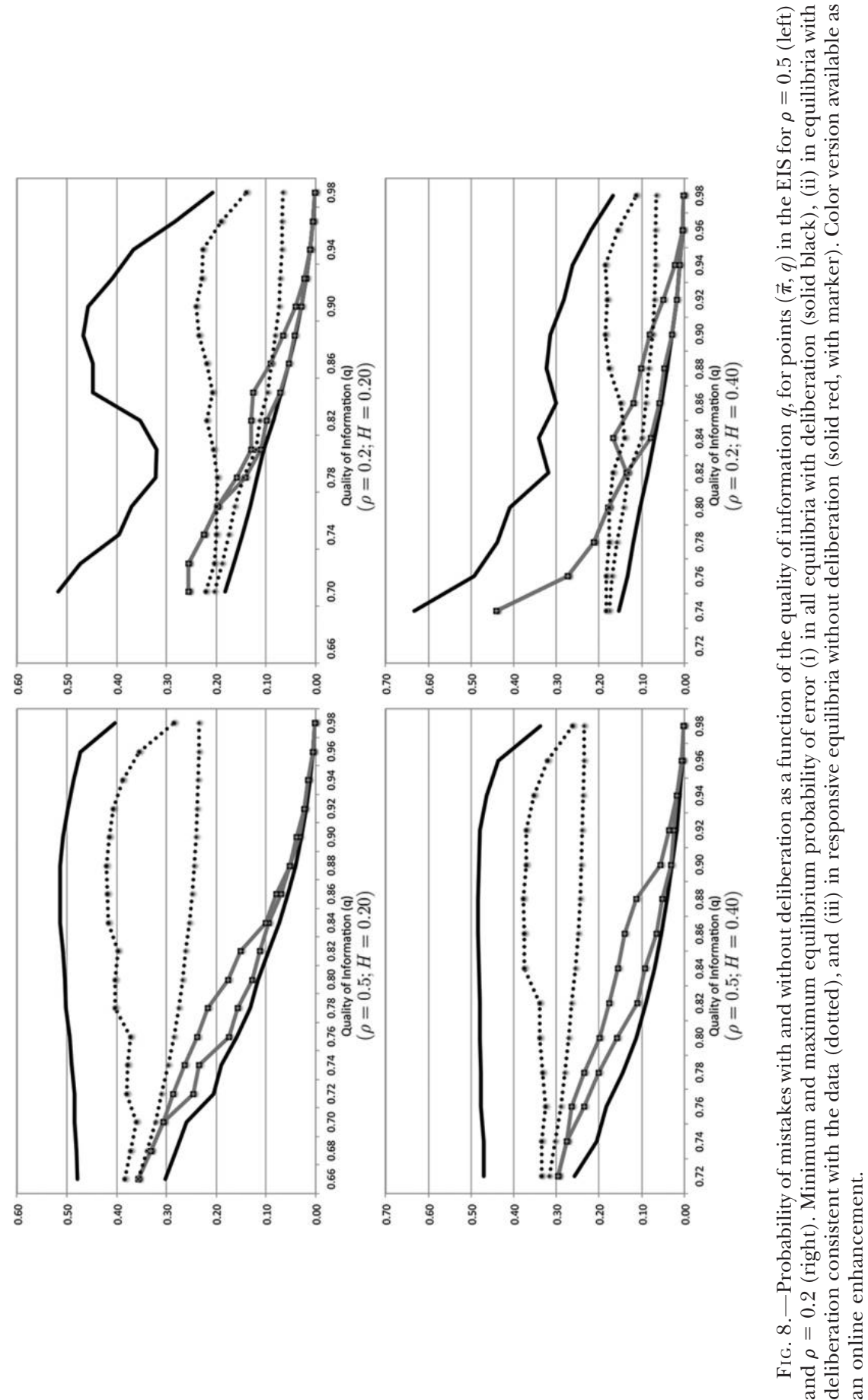

This content downloaded from 131.215.071.079 on April 18, 2018 09:08:51 AM 
by the confidence set $C S$ (see sec. B in the supplemental appendix for details on how we construct $C S$ ). Figure A.4 in the supplemental appendix reproduces figure 7 for all points in the confidence set. Our main conclusion remains unaltered: deliberation can be useful when judges' preferences are heterogeneous but will generally be either ineffectual (if we consider all equilibria with deliberation) or counterproductive (for equilibria consistent with the data) when the court is relatively homogeneous. A similar observation holds for the results in figure 8 .

Nonresponsive equilibria.-In the results above, we compared the probability of incorrect decisions in equilibria with deliberation with the corresponding probability of mistakes in responsive Nash equilibria of the voting game without communication. In some points in the EIS, however, the voting game without deliberation admits only unresponsive BNE, where the decision of the court does not depend on judges' private information (i.e., at least two judges vote unconditionally to overturn or uphold). In these unresponsive BNE, the minimum and maximum probabilities of error equal $\min \{\rho, 1-\rho\}$ and $\max \{\rho, 1-\rho\}$, respectively. We should therefore keep in mind that in addition to any positive effect deliberation can have on outcomes relative to responsive equilibria of voting without deliberation, prevote communication also expands the set of court configurations for which equilibrium outcomes are responsive to private information. Indeed, in close to 14 percent of points in the EIS for $\rho=0.5$ and 8 percent of points in the EIS for $\rho=0.2$, private information is too imprecise to overcome differences in preferences, and the only equilibrium of voting without deliberation is completely unresponsive to judges' private signals.

Efficient deliberation.- The results so far are agnostic about equilibrium selection. It could be argued, however, that equilibria that maximize judges' aggregate welfare constitute a focal point, both in the game with deliberation and in the game without deliberation. If this were the case, deliberation could in fact improve welfare and would certainly do so if we do not restrict to equilibria consistent with the data. In order to quantify this potential gain, we adopt a utilitarian approach and compare social welfare in the equilibria that maximize the sum of judges' payoffs with and without deliberation, for equilibria consistent with the data and all equilibria. (We report the details of this exercise in the supplemental appendix, sec. E.) The results confirm our previous findings. When we consider the maximum aggregate welfare for points in the EIS for $\rho=0.5$ across all equilibria, we find that the gain from efficient deliberation is fairly small and concentrated at higher levels of competence and preference heterogeneity. Restricting to efficient equilibria consistent with the data, instead, deliberation generally reduces welfare (this is consistent with our previous results for the EIS with $\rho=0.5$ ). In fact, for relatively homogeneous courts $(H \leq 0.5)$, aggregate welfare at the efficient equilibrium 
without deliberation for a moderate level of ability of $q=0.80$ exceeds aggregate welfare at the efficient equilibrium with deliberation at $q=$ 0.90 .

Refining the identified set via instrumental variables.-Our treatment of deliberation thus far has been purposely agnostic and imposes only the weak requirement that judges be playing a communication equilibrium. As a result the identified set of parameters can be large. In this section, we refine the identified set using an instrument: an exogenous case characteristic $Z$ that affects vote outcomes indirectly, through its effect on deliberation, but does not change the structural parameters $(\rho, \vec{\pi}, \vec{q})$. The variable $Z$ is an instrument in the sense that it affects the endogenous vote outcomes but does not affect the structural parameters that characterize judges' preferences and the information structure. ${ }^{26}$

The availability of the instrument $Z$ introduces additional constraints to the model, which shrinks the identified set of model parameters. Let $\vec{p}_{v}(z)$ be the conditional distribution of the voting profiles given $Z=z$. Then for every $z$, the incentive compatibility constraints (eqq. [2]-[4]) and the equilibrium conditions (eq. [6]) with $\mu(\vec{v} \mid \vec{t})$ replaced by $\mu(\vec{v} \mid \vec{t}, z)$ and $\vec{p}_{v}$ replaced by $\vec{p}_{v}(z)$ hold. They form the additional identifying restrictions for $(\rho, \vec{\pi}, \vec{q})$. These additional restrictions are not redundant precisely because $Z$ affects deliberation effectiveness and thus varies the equilibrium voting profile distribution.

As an instrument we use here the variable Caseload, defined as the number of cases per judge in a given year in a given circuit. The caseload of a circuit directly influences the time constraint on the deliberation of the cases in that circuit and, as a result, the extent and effectiveness of deliberation. Because Caseload ultimately depends on the number of appealed cases and the number of appellate judges at the circuit levelboth of which are exogenous and predetermined from the judges' point of view - it can be reasonably believed to be exogenous to judges' beliefs, biases, and signal quality. Likewise, it seems eminently plausible that time constraints on deliberation will not affect judges' prior beliefs and preference parameters. Here, we proceed under the assumption that Caseload is exogenous to all the model parameters. In the supplemental appendix, section $\mathrm{F}$, we show that these results are robust even after allowing the possibility that Caseload may affect judges' signal quality $q$ (for instance, a high Caseload may force judges to make more hasty decisions).

Using Caseload as our instrument, we can refine the identified set using the idea of "intersection bounds" (as in Chernozhukov, Lee, and Rosen [2013]). Specifically, we separately estimate identified sets conditional on seven values of Caseload, corresponding to seven quantiles (0.125, 0.25,

\footnotetext{
${ }^{26}$ In contrast to the regression context, in which the requirements of an instrument variable are well understood, in our moment inequality model with partial identification, using Caseload as an instrument requires that the model parameters do not depend on it.
} 
$0.375,0.5,0.625,0.75,0.875)$ of the variable in the data. Then we can intersect all these sets (as well as the estimated identified set reported earlier) to obtain our final estimate of the identified set of model parameters.

The procedure is illustrated in figure A.5 in the supplemental appendix. We find that the intersection procedure shrinks the identified set substantially. Specifically, the number of grid points in the original estimated sets was 38,963 and shrinks to 13,912 (roughly one-third) points once we perform the intersection with the identified sets conditional on these seven percentiles of the caseload variable. Figure A.5 plots projections of the EIS for $q=0.76$ and $\rho=0.5$ at three different levels of intersection. For the plots here, we see that the shrinking procedure asymmetrically eliminates points from the identified set; particularly, comparing the level 0 to level 3 figures, we see that the latter no longer includes points where $\pi_{1}, \pi_{2}, \pi_{3}<0.4$. Thus, these intersection bounds suggest that the data are consistent with courts composed of judges who are less predisposed to overturn.

For the refined identified set, we have also recomputed the graphs illustrating the probability of mistakes with and without deliberation, as shown in figure A.6 in the supplemental appendix. As the figure shows, the refinement of the EIS does not change our previous results regarding the effect of deliberation. The reason is that shrinking the identified set, while eliminating courts very prone to overturning, has not removed highly homogeneous courts, for which deliberation is least beneficial relative to no deliberation.

Unanimous decisions.-Around 90 percent of the cases in our data set were decided by unanimous decisions of the judges. This may raise worries regarding identification, especially as typical empirical strategies for estimation of the spatial voting model rely solely on divided votes (see Poole and Rosenthal 1985; Heckman and Snyder 1997; Clinton et al. 2004). Our empirical approach, however, is based on matching moments describing the frequency of votes of all types. As a result, all voting outcomes, including the unanimous ones, provide information for our identification.

To demonstrate the fact that the unanimous votes provide useful variation, we did the following experiment. In this experiment, we remove the unanimous voting profiles (000 and 111) from the benchmark probability distribution of the voting profile $\hat{p}$ to obtain the following artificial voting profile distribution:

$$
\hat{p}^{\mathrm{dissent}}=(.00, .20, .13, .25, .15, .18, .00, .09)^{\prime} .
$$

With this artificial distribution replacing the actual empirical distribution $\hat{p}$, we reestimate the identified set of the structural parameters and the error probabilities and compare them with those originally obtained. Because $\hat{p}$ and $\hat{p}^{\text {dissent }}$ imply the same conditional probability distribution 
for the dissenting voting profiles given that votes are not unanimous, we should expect the estimates based on these two to be similar were it true that the identification comes solely from divided votes. However, we find that the estimates based on $\hat{p}$ and those based on $\hat{p}^{\text {dissent }}$ are rather different. The difference is apparent, for example, in the estimates of the max/ min error probabilities consistent with the data (see fig. A.7 in the supplemental appendix). In particular, the estimated error bounds using the artificial data are much lower and do not generally overlap with those using the actual data.

The reason that the unanimous votes are important for us lies in the basic structure of our identification strategy. In particular, our identification uses the conditional voting profile distribution (given $X=x$ ) to back out the value of the structural parameters (biases and quality of information of judges). Naturally, the whole voting profile distribution matters. $^{27}$

Common values revisited: questions of law and fact.-A fundamental aspect of our common-values model is our understanding of what constitutes an error by the appellate court, which we define by whether it rightly or wrongly determines that the law was applied correctly in the trial court. Here we discuss this assumption and provide a robustness exercise that focuses on a restricted sample of cases.

A key distinction in the process by which appellate judges determine if the lower court made one or more mistakes is whether the question is one of law or fact. Appellate judges give great deference to trial courts on questions of fact. For example, findings of fact are reviewed under a clear error standard. ${ }^{28}$ This also applies, for the most part, in cases involving jury instructions. ${ }^{29}$ Questions of law are reviewed de novo by the appellate courts. ${ }^{30}$ However, this standard is less lax than de novo would

\footnotetext{
${ }^{27}$ One partial analogy with the structural empirical auction literature to note is that estimating an auction model using the "number of actual bidders" vs. the "number of potential bidders" (with the difference between the two being the bidders who do not participate because their bid would be below the reserve price) can lead to very different estimates.

${ }^{28}$ See, e.g., United States v. Rodgers, 656 F.3d 1023, 1026 (9th Cir. 2011) (motion to suppress); United States v. Stoterau, 524 F.3d 988, 997 (9th Cir. 2008) (sentencing); United States v. Doe, 136 F.3d 631, 636 (9th Cir. 1998) (bench trial).

29 "In reviewing jury instructions, the relevant inquiry is whether the instructions as a whole are misleading or inadequate to guide the jury's deliberation" (United States v. Dixon, 201 F.3d 1223, 1230 [9th Cir. 2000]). The formulation of instructions, whether or not to include special verdict forms, whether the record is sufficient to warrant a lesser-included offense charge, etc., are all reviewed for abuse of discretion. More purely legal questions (such as whether the charge omits or misstates a material element of the crime) are reviewed de novo. However, given the rather black and white nature of whether or not the trial court was substantively right or wrong in listing the elements of the offense in the charge to the jury, jury instructions produce well-defined errors in a full information environment.

${ }^{30}$ For instance, claims of insufficient evidence are reviewed under this more lenient standard; see United States v. Bennett, 621 F.3d 1131, 1135 (9th Cir. 2010); United States v. Sullivan, 522 F.3d 967, 974 (9th Cir. 2008).
} 
imply. In particular, the appellate court does not determine whether the evidence at the trial established guilt beyond a reasonable doubt. Instead, the relevant question is whether, after viewing the evidence in the light most favorable to the prosecution, "any rational trier of fact" could find the defendant guilty. ${ }^{31}$ This makes the standard of review far more deferential to the lower court and limits the effect of differences in judges' personal evidentiary threshold requirements.

The type of cases for which the common-value assumption may be considered questionable is admissibility. While a trial court's decision to admit evidence is generally reviewed for abuse of discretion, the substantive law that governs searches and seizures has not been stable during the years covered in the data. ${ }^{32}$ Thus, while in admissibility cases appellate courts also "determine whether or not the law was applied correctly in the trial court," the assumption that errors are well defined given knowledge of all relevant facts and law, while still plausible, is less straightforward in this context. Because of this, in a robustness exercise we reestimate our model and counterfactuals focusing exclusively on sentencing and sufficiency cases. We find that our main conclusions are qualitatively unchanged (see the supplemental appendix, sec. G).

\section{Conclusion}

Deliberation is ubiquitous in collective decision making. What is less clear is whether talking can have an effect on what people actually do. In this paper, we quantify the effect of deliberation on collective choices. To do this we structurally estimate a model of voting with deliberation, allowing us to disentangle committee members' preferences, information, and strategic considerations and, ultimately, to compare equilibrium outcomes under deliberation with a counterfactual scenario in which prevote communication is precluded.

Because the structural parameters characterizing judges' biases and quality of information are only partially identified, we obtain confidence regions for these parameters using a two-step estimation procedure that allows flexibly for characteristics of the alternatives and the individuals. We find that deliberation can be useful when judges tend to disagree ex

31 See Jackson v. Virginia, 443 U.S. 307, 319 (1979).

32 Federal courts, for instance, generally did not apply Fourth Amendment concerns against the states until 1961 (Mapp v. Ohio, 367 U.S. 643 [1961]), and search and seizure cases were long contested even at the Supreme Court, so there was more room for judicial ideology to affect decisions at the circuit courts given the unsettled nature. Fifth Amendment concerns (notably Miranda v. Arizona, 384 U.S. 436 [1966]) also developed new law. Further, many investigatory tactics are reviewed de novo by intermediate courts: Terry stops (United States v. Grigg, 498 F.3d 1070, 1074 [9th Cir. 2007]), warrantless searches, seizures, and entries are treated similarly, e.g., United States v. Franklin, 603 F.3d 652, 655 (9th Cir. 2010). 
ante and their private information is relatively imprecise; otherwise, it tends to reduce the effectiveness of the court. These findings extend the reach of previous theoretical results and complement findings from laboratory experiments.

Our analysis may be extended in various ways. In this paper, we have been largely agnostic regarding the specific communication protocols used in US appellate courts, and we focused on communication equilibria because the set of outcomes induced by communication equilibria coincides with the set of outcomes induced by sequential equilibria of any possible communication sequence. In other committee voting settings, however, we may be able to restrict attention to a particular communication protocol; in those cases, equilibrium analysis may yield more precise predictions, which would allow us to further tighten the identified set of parameters and predictions about the effects of deliberation. This we leave for future explorations.

\section{References}

Austen-Smith, D., and J. S. Banks. 1996. "Information Aggregation, Rationality, and the Condorcet Jury Theorem." American Polit. Sci. Rev. 90:34-45.

Austen-Smith, D., and T. Feddersen. 2005. "Deliberation and Voting Rules." In Social Choice and Strategic Decisions: Essays in Honor of Jeffrey S. Banks, edited by D. Austen-Smith and J. Duggan, 269-316. Berlin: Springer.

. 2006. "Deliberation, Preference Uncertainty, and Voting Rules." American Polit. Sci. Rev. 100:209-18.

Beresteanu, A., I. Molchanov, and F. Molinari. 2011. "Sharp Identification Regions in Models with Convex Moment Predictions." Econometrica 79:17851821.

Berry, S., and E. Tamer. 2006. "Identification in Models of Oligopoly Entry." In Advances in Economics and Econometrics: Theory and Applications, Ninth World Congress, edited by R. Blundell, W. K. Newey, and T. Persson, 46-85. Cambridge: Cambridge Univ. Press.

Cameron, A. C., J. B. Gelbach, and D. L. Miller. 2011. "Robust Inference with Multiway Clustering." I. Bus. and Econ. Statis. 29:238-49.

Cantillon, E., and M. Pesendorfer. 2006. "Combination Bidding in Multi-unit Auctions." Working paper, London School Econ.

Chernozhukov, V., H. Hong, and E. Tamer. 2007. "Estimation and Confidence Regions for Parameter Sets in Econometric Models." Econometrica 75:1234-75.

Chernozhukov, V., S. Lee, and A. M. Rosen. 2013. "Intersection Bounds: Estimation and Inference." Econometrica 81:667-737.

Clinton, J. D., S. Jackman, and D. Rivers. 2004. "The Statistical Analysis of Roll Call Data." American Polit. Sci. Rev. 55:355-70.

Coughlan, P. 2000. "In Defense of Unanimous Jury Verdicts: Mistrials, Communication, and Strategic Voting." American Polit. Sci. Rev. 94:375-93.

Degan, A., and A. Merlo. 2009. "Do Voters Vote Ideologically?" I. Econ. Theory 144:1869-94.

De Paula, A., and A. Merlo. 2009. "Identification and Estimation of Preference Distributions When Voters Are Ideological.” Working paper, Univ. Pennsylvania. 
De Paula, A., and X. Tang. 2012. "Inference of Signs of Interaction Effects in Simultaneous Games with Incomplete Information.” Econometrica 80:143-72.

Dickson, E., C. Hafer, and D. Landa. 2008. "Cognition and Strategy: A Deliberation Experiment." I. Politics 70:974-89.

Doraszelski, U., D. Gerardi, and F. Squintani. 2003. "Communication and Voting with Double-Sided Information.” Contributions Theoretical Econ. 3:1-39.

Feddersen, T., and W. Pesendorfer. 1997. "Voting Behavior and Information Aggregation in Elections with Private Information.” Econometrica 65:1029-58.

_ 1998. "Convicting the Innocent: The Inferiority of Unanimous Jury Verdicts under Strategic Voting." American Polit. Sci. Rev. 92:23-35.

Fischman, J. B. 2011. "Estimating Preferences of Circuit Judges: A Model of Consensus Voting." L. Law and Econ. 54:781-809.

—. 2015. "Interpreting Circuit Court Voting Patterns: A Social Interactions Framework." L. Law, Econ., and Org. 31 (4): 808-42.

Forges, F. 1986. "An Approach to Communication Equilibria." Econometrica 54 (6): 1375-85.

Galichon, A., and M. Henry. 2011. "Set Identification in Models with Multiple Equilibria." Rev. Econ. Studies 78 (4): 1264-98.

Gerardi, D., and L. Yariv. 2007. "Deliberative Voting." L. Econ. Theory 134:317-38.

Goeree, J., and L. Yariv. 2011. "An Experimental Study of Collective Deliberation." Econometrica 79 (3): 893-921.

Gole, T., and S. Quinn. 2014. "Committees and Status Quo Bias: Structural Evidence from a Randomized Field Experiment.” Discussion Paper no. 733, Dept. Econ., Univ. Oxford.

Grieco, P. 2014. "Discrete Games with Flexible Information Structures: An Application to Local Grocery Markets." RAND I. Econ. 45:303-40.

Guarnaschelli, S., R. McKelvey, and T. Palfrey. 2000. "An Experimental Study of Jury Decision Rules.” American Polit. Sci. Rev. 94:407-23.

Hansen, S., M. McMahon, and C. Velasco Rivera. 2013. "Preferences or Private Assessments on a Monetary Policy Committee?” Working paper, Univ. Pompeu Fabra.

Heckman, J., and J. J. Snyder. 1997. "Linear Probability Models of the Demand for Attributes with an Empirical Application to Estimating the Preferences of Legislators." In "Special Issue in Honor of Richard E. Quandt," RAND I. Econ. 28:S142-S189.

Henry, M., and I. Mourifie. 2013. "Euclidean Revealed Preferences: Testing the Spatial Voting Model." L. Appl. Econometrics 28:650-66.

Iaryczower, M., G. Katz, and S. Saiegh. 2013. "Voting in the Bicameral Congress: Large Majorities as a Signal of Quality.” L. Law, Econ. and Org. 29 (5): 957-91.

Iaryczower, M., G. Lewis, and M. Shum. 2013. "To Elect or to Appoint? Bias, Information, and Responsiveness of Bureaucrats and Politicians." L. Public Econ. 97:230-44.

Iaryczower, M., and M. Shum. 2012a. "Money in Judicial Politics: Individual Contributions and Collective Decisions." Manuscript, Princeton Univ.

- 2012b. "The Value of Information in the Court: Get It Right, Keep It Tight." A.E.R. 102:202-37.

Kamenica, E., and M. Gentzkow. 2011. "Bayesian Persuasion.” A.E.R. 101 (6): 2590-2615.

Kawai, K., and Y. Watanabe. 2013. "Inferring Strategic Voting." A.E.R. 103 (2): 624-62.

Landa, D., and A. Meirowitz. 2009. "Game Theory, Information, and Deliberative Democracy.” American I. Polit. Sci. 53 (2): 427-44. 
Li, H., S. Rosen, and W. Suen. 2001. "Conflicts and Common Interests in Committees." A.E.R. 91:1478-97.

Lizzeri, A., and L. Yariv. 2011. "Sequential Deliberation.” Working paper, New York Univ.

Londregan, J. 1999. “Estimating Legislators' Preferred Points.” Polit. Analvsis 8 (1): $35-56$.

Martin, A., and K. Quinn. 2002. "Dynamic Ideal Point Estimation via Markov Chain Monte Carlo for the US Supreme Court, 1953-1999." Polit. Analvsis 10 (2): $134-53$.

. 2007. "Assessing Preference Change on the US Supreme Court." I. Law. Econ. and Org. 23 (2): 365-85.

McCubbins, M., and B. Rodriguez. 2006. "When Does Deliberating Improve Decision-Making?" J. Contemporary Legal Studies 15:9-50.

Meirowitz, A. 2006. "Designing Institutions to Aggregate Preferences and Information." O. I. Polit. Sci. 1:373-92.

Menzel, K. 2011. "Robust Decisions for Incomplete Structural Models of Social Interactions." Working paper, New York Univ.

Myerson, R. B. 1986. "Multistage Games with Communication." Econometrica 54 (2): 323-58.

Poole, K., and H. Rosenthal. 1985. "A Spatial Model for Legislative Roll Call Analysis.” American I. Polit. Sci. 29:357-84.

78

Ryan, S. 2012. "The Costs of Environmental Regulation in a Concentrated Industry.” Econometrica 80:1019-61.

Shi, X., and M. Shum. 2015. "Simple Two-Stage Inference for a Class of Partially Identified Models." Econometric Theory 31:493-520.

Songer, D. R. 2008. "United States Courts of Appeals Database." Judicial Research Initiative, Univ. South Carolina.

Stasser, G., and W. Titus. 1985. "Pooling of Unshared Information in Group Decision Making: Biased Information Sampling during Discussion.” L. Personalit and Soc. Psvchology 48 (6): 1467-78.

Sweeting, A. 2009. "The Strategic Timing of Radio Commercials: An Empirical Analysis Using Multiple Equilibria.” RAND J. Econ. 40:710-42.

Wan, Y., and H. Xu. 2010. "Semiparametric Estimation of Binary Decision Games of Incomplete Information with Correlated Private Signals.” Working paper, Pennsylvania State Univ.

Xu, H. 2014. "Estimation of Discrete Games with Correlated Types." Econometrics I. 17 (3): 241-70.

Zuk, G., D. J. Barrow, and G. S. Gryski. 2009. Multi-user Database on the Attributes of United States Appeals Court Judges, 1801-2000. Inter-university Consortium for Political and Social Research (ICPSR) [distributor]. Ann Arbor: Univ. Michigan. 